ANL-HEP-PR-09-94, EFI-09-22, MSUHEP-091025

\title{
Next-to-leading order QCD corrections to s-channel single top quark production and decay at the LHC
}

\author{
Sarah Heim,,$*$ Qing-Hong Cao, ${ }^{2,3,+}$ Reinhard Schwienhorst, ${ }^{1,+}$ and C.-P. Yuan ${ }^{1, \$}$ \\ ${ }^{1}$ Department of Physics \& Astronomy, \\ Michigan State University, East Lansing, MI, 48824, USA \\ ${ }^{2}$ High Energy Physics Division, Argonne National Laboratory, Argonne, IL, 60439, USA \\ ${ }^{3}$ Enrico Fermi Institute, University of Chicago, Chicago, IL, 60637, USA
}

\begin{abstract}
We present a study of electroweak production of top and antitop quarks in the s-channel mode at the LHC, including next-to-leading order (NLO) quantum chromodynamics (QCD) corrections to the production and decay of the single (anti)top quark. The spin is preserved in production and decay by using the narrow width approximation for the (anti)top quark. We show the effect of different $O\left(\alpha_{s}\right)$ contributions on the inclusive cross section and various kinematic distributions at parton level after imposing relevant kinematic cuts to select s-channel single top quark events. We also discuss several possibilities for measuring the top quark polarization.
\end{abstract}

*Electronic address: heimsara@msu.edu

${ }^{\dagger}$ Electronic address: caoq@hep.anl.gov

${ }^{\ddagger}$ Electronic address: schwier@pa.msu.edu

${ }^{\S}$ Electronic address: yuan@pa.msu.edu 


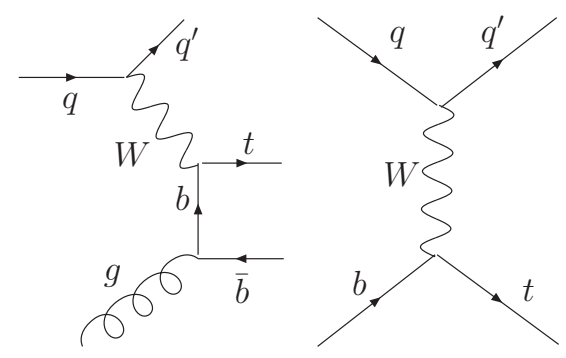

(a)

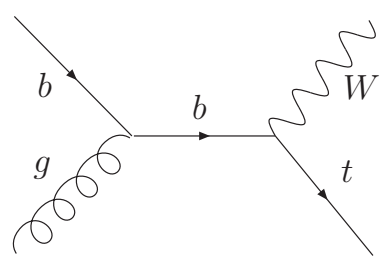

(b)

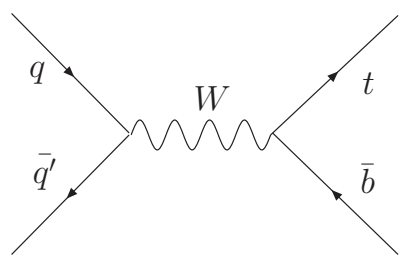

$(c)$

Figure 1: Representative Feynman diagrams of the three single top quark production modes: tchannel (a), associated production (b) and s-channel (c).

\section{INTRODUCTION}

Recent results at the Tevatron $p \bar{p}$ collider have confirmed the existence of electroweak single top quark production [1-5]. While the Tevatron can be considered a $t \bar{t}$ factory, measurements of single top quark properties are statistics limited. This limitation does not exist at the LHC. The main mode of top quark production at the LHC is still strong interaction top quark pair production, but the number of produced single top quark events will be large enough for precision measurements.

Single top quark events are of considerable importance for probing the Standard Model of particle physics (SM). As the top quark decays via the weak interaction before it can hadronize, it is possible to measure its polarization. In single top quark events, the top quark is coupled to the bottom quark with an amplitude proportional to the CabibboKobayashi-Maskawa (CKM) matrix element $V_{t b}$, so that a value for $V_{t b}$ can be obtained by measuring the single top quark production cross section.

Electroweak single top quark production at the LHC occurs in three different modes (cf. Fig. 11). The process with the largest cross section is the t-channel exchange of a virtual $W$ boson $\left(b q \rightarrow t q^{\prime}\right.$ and $\left.b \bar{q}^{\prime} \rightarrow t \bar{q}\right)$, also referred to as $W$-gluon fusion, followed by associated production of a top quark and a $W$ boson $\left(b g \rightarrow t W^{-}\right)$and the s-channel decay of a virtual $W$ boson $\left(q \bar{q}^{\prime} \rightarrow W^{*} \rightarrow t \bar{b}\right)$.

The top quark is the heaviest known elementary particle, which makes it an excellent candidate for new physics searches. The cross sections of the three single top quark production modes are sensitive to different kinds of new physics [6-25]. The single top quark s-channel 
cross section is especially sensitive to additional bosons, which makes it a very important channel in spite of its comparably small cross section. The t-channel provides good means to search for flavor changing neutral currents (FCNC) and the associated production cross section changes with a modified $W t b$ or $W q q^{\prime}$ coupling. Both t-channel and $W t$ associated production are sensitive to the bottom quark parton distribution function (PDF). A detailed knowledge of the properties of single top quark production is necessary, as it is an important background for several Higgs boson production modes. The $W t$ associated production serves as background to Higgs boson searches in the decay channel $H \rightarrow W W[26]$ and also to processes involving a charged Higgs boson like $b g \rightarrow t H^{ \pm}$and $H^{ \pm} \rightarrow \tau \nu$ [27].

In order to extract the single top quark signal from the large QCD and $W+j e t s$ backgrounds, but also in cases in which single top quark production is a background itself, accurate theoretical predictions including higher order QCD corrections are needed. The NLO QCD corrections to the single top quark production have been carried out in Refs. [28 37]. Furthermore, the complete NLO QCD calculations including both single top quark production and decay have been carried out for the Tevatron in several studies [38 43]. To a lesser extend, single top quark production is also affected by electroweak corrections and possibly virtual supersymmetric effects [44 46]. In this paper we use the full NLO QCD calculations for single top quark production at the LHC in order to present a detailed phenomenological analysis, focusing on signal cross sections and kinematical distributions at parton level after imposing simple kinematic cuts.

In contrast to the Tevatron, the LHC is a $p p$ collider and we have to analyze top and antitop quark production separately. In this paper, we present distributions for top quark production alone where the top-antitop quark differences are small and contrast them with the results for the antitop quark where they are not.

In Sec. II, we first present the inclusive cross section for s-channel single top quark production and discuss its dependence on the center of mass energy of the collider $\left(E_{c . m .}\right)$, the top quark mass $\left(m_{t}\right)$ and renormalization and factorization scales. We also evaluate PDF uncertainties. In Sec. III, we examine the effect of various kinematic cuts. Kinematical distributions of final state objects and spin correlations are discussed in Sec. IV. Our conclusion follows in Sec. V] 


\section{CROSS SECTION (INCLUSIVE RATE)}

In this section, we show the inclusive production rates for s-channel single top quark production and discuss their dependence on $E_{c . m .}, m_{t}$ and factorization and renormalization scales. We present numerical results for s-channel single top quark events considering the leptonic decay of the $W$ boson from the top quark decay at the LHC (a $p p$ collider). Unless otherwise specified, we use the NLO parton distribution function (PDF) set CTEQ6.6M [47]51], defined in the $\overline{M S}$ scheme, and the NLO (2-loop) running coupling $\alpha_{s}$ with $\Lambda_{\overline{M S}}$ provided by the PDFs. For the CTEQ6.6M PDFs, $\Lambda_{\overline{M S}}^{(4)}=0.326 \mathrm{GeV}$ for four active quark flavors. For the numerical evaluation, we choose the following set of SM input parameters: $G_{\mu}=$ $1.16637 \times 10^{-5} \mathrm{GeV}^{-2}, M_{W}=80.413 \mathrm{GeV}, M_{Z}=91.187 \mathrm{GeV}$, and $\alpha_{s}\left(M_{Z}\right)=0.1186$. The square of the weak gauge coupling is $g^{2}=4 \sqrt{2} M_{W}^{2} G_{\mu}$.

In this study we focus on the electron leptonic decay of the $W$ boson from the top quark only, but for muon leptons the analysis procedure would be analogue. Including the $O\left(\alpha_{s}\right)$ corrections to $W \rightarrow \bar{q} q^{\prime}$, the branching ratio for the decay of the $W$ boson into leptons is $\operatorname{Br}\left(W \rightarrow l^{+} \nu\right)=0.108$ [52]. If not otherwise specified, the top quark mass is chosen to be $m_{t}=175 \mathrm{GeV}$ [53], the center of mass energy of the collisions $E_{c . m} .=14 \mathrm{TeV}$ and we will choose the renormalization scale $\left(\mu_{R}\right)$ as well as the factorization scale $\left(\mu_{F}\right)$ to be equal to $m_{t}$. In the current section we present inclusive cross sections, which include all $W$ boson decay modes.

In order to calculate NLO QCD differential cross sections we adopt the one-cutoff phase space slicing (PSS) method [54 56] with a cutoff parameter $s_{\min }=5 \mathrm{GeV}^{2}$.

\section{A. Inclusive cross section}

As in our previous studies [40, 41], we divide the higher-order QCD corrections into three separate gauge invariant sets: corrections to the initial particles (INIT), corrections to the final state (FINAL), and corrections to the top quark decay (SDEC). The explicit diagrams and definitions for the different corrections can be found in Ref. [39]. For $E_{c . m .}=$ $14 \mathrm{TeV}$ and $m_{t}=175 \mathrm{GeV}$, Table \ shows the inclusive cross sections for top and antitop quark production as well as the individual $O\left(\alpha_{s}\right)$ contributions. The effects of the finite widths of top quark and $W$ boson have been included. The total NLO s-channel single top 


\begin{tabular}{lcccc}
\hline \hline & \multicolumn{2}{c}{ Top } & \multicolumn{2}{c}{ Antitop } \\
& Cross section & Fraction of & Cross section & Fraction of \\
& {$[\mathrm{pb}]$} & NLO $(\%)$ & {$[\mathrm{pb}]$} & NLO $(\%)$ \\
\hline Born level & 4.42 & 72.55 & 2.70 & 72.07 \\
\hline INIT & 1.18 & 19.38 & 0.73 & 19.48 \\
FINAL & 0.80 & 13.16 & 0.51 & 13.57 \\
SDEC & -0.31 & -5.09 & -0.19 & -5.11 \\
\hline$O\left(\alpha_{s}\right)$ sum & 1.67 & 27.44 & 1.04 & 27.93 \\
\hline NLO & 6.09 & 100 & 3.74 & 100 \\
\hline \hline
\end{tabular}

Table I: Inclusive single top quark production cross sections for different subprocesses, for top quark production (left) and antitop quark production (right). $E_{c . m .}=14 \mathrm{TeV}$ and $m_{t}=175 \mathrm{GeV}$.

quark production cross section agrees with Ref. [28], but updated values for the electroweak parameters are used.

As can be seen in Table $\llbracket$, the LO cross section for antitop quark production $(\bar{u} d \rightarrow \bar{t} b)$ is $39 \%$ smaller than the cross section for top quark production $(u \bar{d} \rightarrow t \bar{b})$. This is due to the difference in parton densities of the colliding protons. While in both cases the antiquark is from the quark sea of one of the incoming protons, the probability that it collides with an up quark from the other proton is higher than the probability for a collision with a down quark.

The $O\left(\alpha_{s}\right)$ corrections increase the cross section by $38 \%$ for both top and antitop quark production. The largest contribution to the $O\left(\alpha_{s}\right)$ corrections comes from the initial state, due to the enhancement of collinear physics and the large phase space for additional parton radiation. As expected, and as also observed at the Tevatron, the correction to the top quark decay is small [40, 41].

The obtained inclusive cross sections at the LHC for s-channel single top quark events (considering the $W$ boson decay branching ratio and using $m_{t}=175 \mathrm{GeV}, 172.5 \mathrm{GeV}$ and $170 \mathrm{GeV}$ ) are shown in Table $\Pi$ and Fig. 2 for top and antitop quark production at different $E_{c . m .}$. For the LHC injection energy, the cross sections become very small, for $m_{t}=175 \mathrm{GeV}$ 


\begin{tabular}{lccc}
\hline \hline & & Top & Antitop \\
$m_{t}[\mathrm{GeV}]$ & $E_{\text {c.m. }}[\mathrm{TeV}]$ & Cross section $[\mathrm{pb}]$ & Cross section $[\mathrm{pb}]$ \\
\hline \multirow{2}{*}{175} & 14 & 6.09 & 3.74 \\
& 10 & 3.96 & 2.28 \\
& 7 & 2.45 & 1.30 \\
\hline \multirow{2}{*}{172.5} & 14 & 6.43 & 3.96 \\
& 10 & 4.19 & 2.42 \\
170 & 7 & 2.58 & 1.38 \\
\hline \hline
\end{tabular}

Table II: Inclusive single top quark production cross sections for top quark production (left) and antitop quark production (right) at $E_{c . m .}=14,10$ and $7 \mathrm{TeV}$ and three different $m_{t}$.

(a)

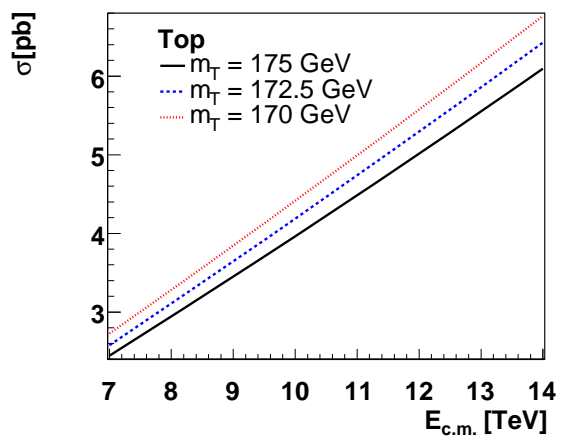

(b)

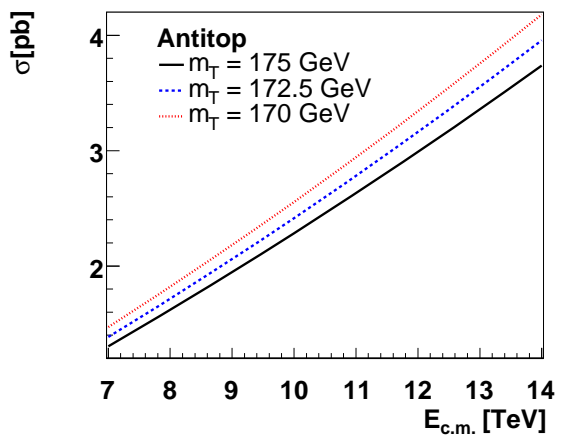

Figure 2: $E_{c . m}$. dependence of the single top (a) and antitop (b) quark production cross section for $m_{T}=175,172.5,170 \mathrm{GeV}$.

and $E_{c . m}=900 \mathrm{GeV}$, the inclusive cross section for top (antitop) quark production is 0.018 (0.005) $\mathrm{pb}$.

For $m_{t}=175 \mathrm{GeV}$, Fig. 3 compares the $E_{c . m}$. dependence of NLO and LO cross sections, for top and antitop quark, and for the charge asymmetry ratio $(\mathcal{R})$. $\mathcal{R}$ is defined as the ratio of top over antitop quark production cross sections. Different from the Tevatron, the single top quark production at the LHC (a $p p$ collider) has a large charge asymmetry, 
(a)

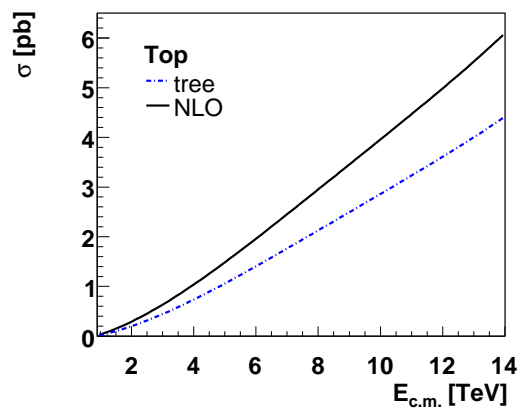

(b)

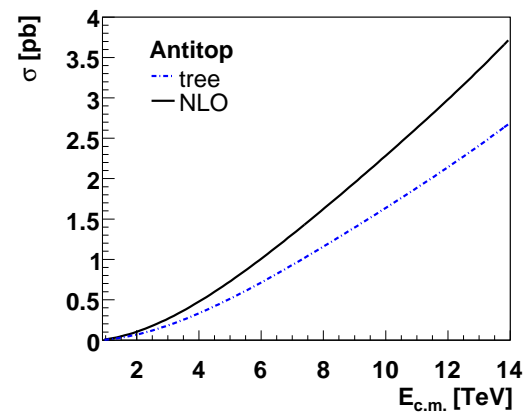

(c)

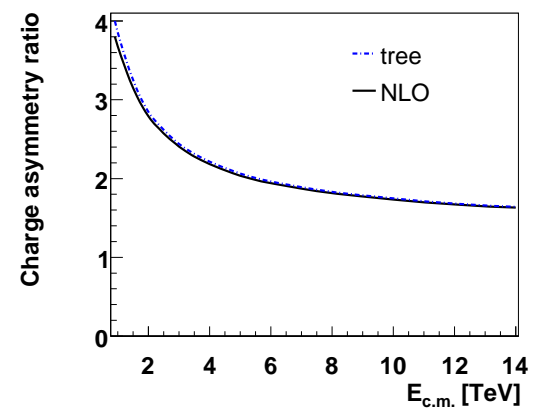

Figure 3: $E_{c . m}$. dependence of single top (a) and antitop (b) quark production cross section at NLO and leading order (LO) for $m_{T}=175 \mathrm{GeV}$ and ratio of cross sections for top/antitop quark production (c).

leading to a difference in the numbers of top versus antitop quarks produced. Such an asymmetry is preserved in the charge of charged leptons from the top quark decay and will be measured at the LHC. It can be seen that for both top and antitop quark, with rising $E_{c . m}$, the cross section grows faster at NLO than at LO. This is mainly due to the higher momentum of the sea quarks and gluons in the colliding protons which increases the initial state corrections. The ratio of top/antitop quark production decreases with higher energy as the PDF difference between up and down quark looses significance.

Without losing generality, in the remaining paper we consider $E_{\text {c.m. }}=14 \mathrm{TeV}$ only.

\section{B. Top quark mass dependence and theoretical uncertainties}

In order to predict the cross section for single top quark production as precisely as possible, we need to understand how it depends on variations of the input parameters, such as $m_{t}$ dependence, scale dependences, and PDF uncertainties.

The Tevatron has accomplished to reduce the uncertainty of the newest world average $m_{t}$ to $1.3 \mathrm{GeV}$ [57], so it is of interest to see how the cross section varies in this range. It can be seen in Fig. 4, that a variation of $m_{t}$ at $175 \mathrm{GeV}$ of $\pm 5 \mathrm{GeV}$ changes the cross section

of single top quark production by about $\mp 10 \%$. For the current world average of $173.1 \pm$ $1.3 \mathrm{GeV}$ [57], the predicted numerical result for the s-channel single top quark production cross section is $6.36 \mathrm{pb} \pm 0.19 \mathrm{pb}$, where the error of $3 \%$ is due to mass uncertainty only. For antitop quark production the calculation yields $3.90 \mathrm{pb} \pm 0.12 \mathrm{pb}$, also with an error of 
(a)

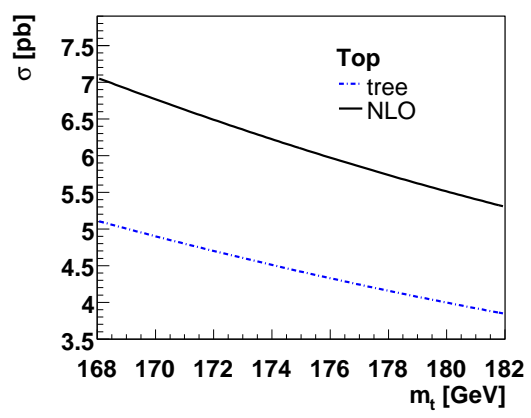

(b)

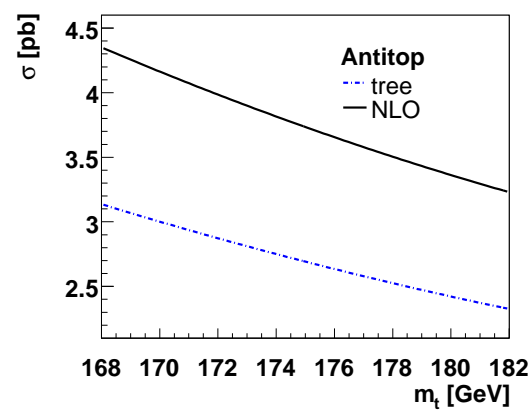

(c)

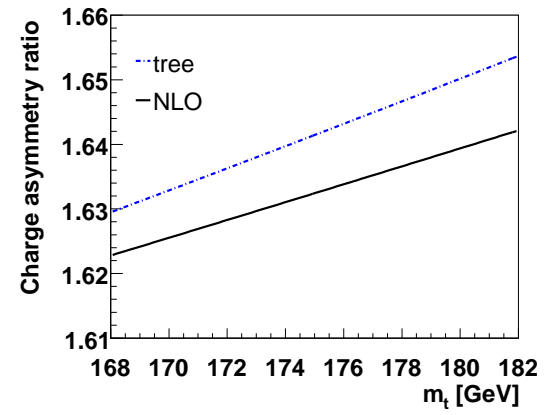

Figure 4: $m_{t}$ dependence of single top (a) and antitop (b) quark production cross section and ratio of cross sections for top/antitop quark production (c), $E_{c . m} .=14 \mathrm{TeV}$.

$3 \%$.

In Fig. 4 (c) the charge asymmetry ratios are $\sim 1.6$ at LO and NLO in the region of $168 \mathrm{GeV}<\mathrm{m}_{\mathrm{t}}<182 \mathrm{GeV}$. Such a large charge asymmetry will be detectable at the LHC. We also note that the ratio curves at LO and NLO both rise with increasing $m_{t}$, which can be understood as follows. If we set $x_{1} \approx x_{2}=x$ where $x_{1,2}$ is the fraction of incoming proton energy carried by the parton, we obtain the average $x$ as $\langle x\rangle \approx m_{t} / \sqrt{s}$ from $\hat{s}=x_{1} x_{2} s$, where $\sqrt{s}=E_{c . m}$. and $\sqrt{\hat{s}}$ is the invariant mass of the incoming partons. The heavier the top quark, the larger $\langle x\rangle$. Since the down quark PDF peaks at lower $x$ value than the up quark PDF, the antitop quark production rate decreases faster than the top quark production rate with increasing $x$ (i.e. increasing $m_{t}$ ). Therefore, it yields the increasing ratio curves in Fig. 4 (c). The $O\left(\alpha_{s}\right)$ corrections, involving more production channels, only distort this picture slightly. For the remainder of this paper we use $m_{t}=175 \mathrm{GeV}$.

Besides $m_{t}$ dependence, single top quark production also suffers from scale dependence, a theoretical uncertainty originated from the unknown higher order corrections. There are two kinds of uncertainties: One is the renormalization scale $\mu_{R}$ which is used for redefining the bare parameters in terms of the renormalized parameters, the other is the factorization scale $\mu_{F}$ which is introduced in order to absorb the collinear divergence into the PDFs. Although both $\mu_{R}$ and $\mu_{F}$ are only introduced for technical reasons and our predictions for the cross section should not depend on them, we see such dependences, as we only work at first order in perturbation theory. In principle, $\mu_{R}$ and $\mu_{F}$ are two independent theoretical parameters. For simplicity, we choose $\mu_{R}=\mu_{F}=\mu_{0}=m_{t}$. We vary $\mu_{0}$ by a factor 2 to estimate the 
(a)

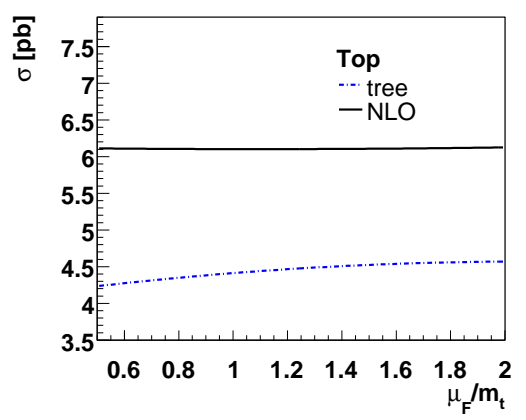

(b)

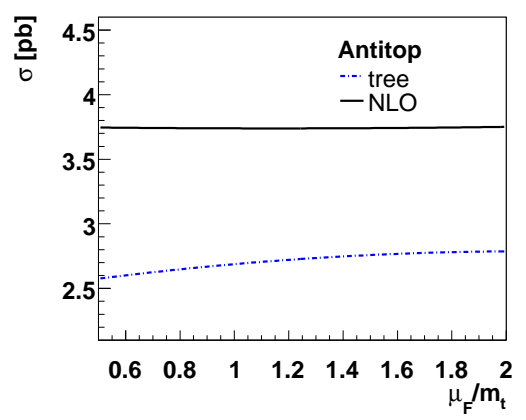

(c)

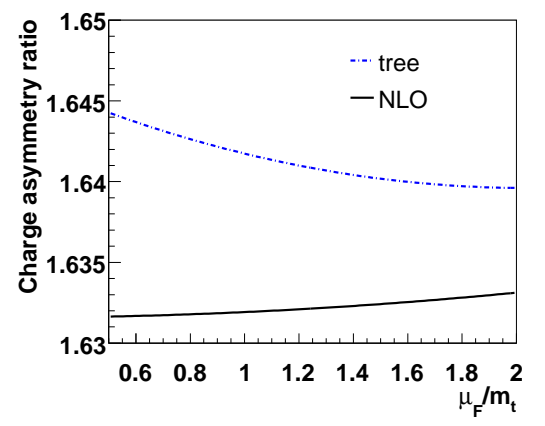

Figure 5: Scale dependence of single top (a) and antitop (b) quark production cross section and ratio of cross sections for top/antitop quark production (c). $E_{c . m}=14 \mathrm{TeV}$ and $m_{t}=175 \mathrm{GeV}$.

size of higher order quantum corrections. Figure 5 (a, b) shows that the $O\left(\alpha_{s}\right)$ corrections reduce the scale dependence of the single top and antitop quark production cross section. In Fig. 5 (c) we see that the charge asymmetry ratio falls at LO with rising $\mu_{F}$, but increases at NLO. This difference comes from additional constituent processes available at NLO.

Another theoretical uncertainty comes from the PDFs. The uncertainty of the CTEQ6.6M PDFs is given in Ref. [51]. At NLO the PDF uncertainty is found to be roughly about $3 \%$ for both top and antitop quark productions. Such a small uncertainty is due to relatively large $x$ values $\left(x \sim m_{t} / \sqrt{s} \sim 0.01\right)$ typical for s-channel single top quark production, where PDF uncertainties are generally small [51].

\section{SINGLE TOP QUARK ACCEPTANCE STUDIES}

The $W$ boson from the top quark can decay into jets or leptons. We only consider the leptonic decay mode as the hadronic decay mode is very difficult to observe experimentally, due to the large QCD background. Thus, the experimental signature of an s-channel single top quark event at NLO is the following: one charged lepton, missing transverse energy $\left(E_{T}\right)$ and two or three jets. As we are discussing single top quark production at parton level here, we only approximate the kinematic acceptance of the detector, and do not consider other detector effects such as b-tagging efficiency or jet energy resolution. For the discussion of the effects of gluon radiation, jets have to be defined as infrared-safe observables. For this study we use the cone-jet algorithm [58], as explained in Ref. [40] with cone-size $R=0.4$ [59]. In this section we furthermore consider $R=1.0$ for reference. The same $R$-separation 


\begin{tabular}{|c|c|c|c|c|c|c|c|c|c|c|c|}
\hline & \multirow[b]{2}{*}{$\sigma[\mathrm{pb}]$} & \multicolumn{5}{|c|}{ Top } & \multicolumn{5}{|c|}{ Antitop } \\
\hline & & $\mathrm{LO}$ & NLO & INIT & FINAL & SDEC & $\mathrm{LO}$ & NLO & INIT & FINAL & SDEC \\
\hline \multirow[t]{2}{*}{ (a) } & $2 \& 3$ jet & 1.42 & 2.02 & 0.57 & 0.19 & -0.16 & 0.90 & 1.28 & 0.37 & 0.12 & -0.10 \\
\hline & 3 jet & & 0.81 & 0.62 & 0.15 & 0.04 & & 0.50 & 0.38 & 0.09 & 0.02 \\
\hline \multirow[t]{2}{*}{ (b) } & $2 \& 3$ jet & 1.19 & 1.69 & 0.45 & 0.17 & -0.12 & 0.75 & 1.08 & 0.29 & 0.11 & -0.07 \\
\hline & 3 jet & & 0.44 & 0.38 & 0.06 & 0.01 & & 0.27 & 0.23 & 0.04 & 0.004 \\
\hline \multirow[t]{2}{*}{ (c) } & $2 \& 3$ jet & 0.68 & 0.79 & 0.21 & 0.02 & -0.13 & 0.44 & 0.51 & 0.14 & 0.01 & -0.08 \\
\hline & 3 jet & & 0.24 & 0.19 & 0.05 & 0.004 & & 0.15 & 0.12 & 0.03 & 0.002 \\
\hline
\end{tabular}

Table III: LO and NLO contributions of cross sections for inclusive two-jet events as well as for exclusive three-jet events, for different cut scenarios. (a) 'loose' cuts with $R_{\text {cut }}=0.4$, (b) 'loose' cuts with $R_{\text {cut }}=1.0$, (c) 'tight' cuts with $R_{\text {cut }}=0.4 . E_{\text {c.m. }}=14 \mathrm{TeV}$ and $m_{t}=175 \mathrm{GeV}$.

is also applied to the separation between leptons and jets.

We consider two sets of kinematic cuts on the final state objects, a 'loose' and a 'tight' cut set [60]:

$$
\begin{gathered}
p_{T}^{\ell} \geq 30 \mathrm{GeV},\left|\eta_{\ell}\right| \leq 2.5, \\
E_{T} \geq 20 \mathrm{GeV}, \\
p_{T}^{j} \geq p_{T}^{\min },\left|\eta_{j}\right| \leq \eta_{j}^{\max }, \\
\Delta R_{\ell j} \geq R_{c u t}, \Delta R_{j j} \geq R_{c u t} .
\end{gathered}
$$

For both sets of cuts, each event is required to have one lepton and at least 2 jets passing all selection criteria. The cut on the separation is chosen to be $R_{c u t}=0.4$ (and for reference 1.0). The 'loose' set of cuts requires the jets to have a transverse momentum of at least $p_{T}^{\text {min }}=30 \mathrm{GeV}$, and a pseudo-rapidity of at most $\eta_{j}^{\max }=5$. At least one of the jets has to come from a $b$ quark. The 'tight' set requires two $b$ jets and all jets to have at least $p_{T}^{\min }=50 \mathrm{GeV}$ and at most $\eta_{j}^{\max }=2.5$.

Table III shows the cross sections for top and antitop quark production in the s-channel single top quark mode, split up into the different LO and NLO contributions after applying the two sets of cuts. For the 'loose' cut set the results are shown for two different values of $R_{\text {cut }}$ and for the 'tight' cut set for $R_{\text {cut }}=0.4$. The results for the 'tight' cuts and $R_{\text {cut }}=1.0$ 
follow the same trend as the other numbers in the table. For inclusive two-jet events and $R_{\text {cut }}=0.4$, the top quark acceptance for the 'loose' cut set is around $33 \%$ both at LO and NLO level, and for the 'tight' cut set around 15\% for the LO contribution and around $13 \%$ for the NLO contributions. For inclusive two-jet events and $R_{c u t}=0.4$, the acceptance for antitop quarks is a bit higher than for top quarks: 34\% at LO and NLO for the 'loose' cut set and $16 \%$ (14\%) for the 'tight' cut set at LO (NLO). The low acceptance is mainly due to the $p_{T}$ cuts on the leptons and jets. The lepton is a product of the $W$ boson decay and its $p_{T}$ distribution peaks under $30 \mathrm{GeV}$, cf. Fig. 7. Imposing a smaller $p_{T}$ cut on the lepton alone would not improve the overall acceptance much because the $p_{T}$ distribution of the $b$ jet produced in association with the (anti)top quark also peaks around $30 \mathrm{GeV}$ (cf. Fig. 9). Hence, a low acceptance still follows from the $p_{T}$ cut on the $b$ jet. A larger value for $R_{c u t}$ reduces the cross section because more events fail the lepton-jet separation cut. For inclusive two-jet events after applying the 'loose' cuts, the reduction from $R_{\text {cut }}=0.4$ to $R_{\text {cut }}=1.0$ is about $17 \%$ for top and antitop quark production, both at LO and NLO level. We further note that the acceptance for antitop quark production is slightly higher than the acceptance for top quark production. This is due to the $\eta$ cuts on the lepton as the lepton in antitop quark production remains in a more central rapidity region than the lepton in top quark production, cf. Fig. 7 . This follows directly from the fact that the top quark is boosted more than the antitop quark. The top quark, when produced, receives large contributions from the valence up quark in the large $x$ regime, therefore it has large momentum along the moving direction of the incoming up quark. Such a boost effect is transferred to the top quark decay products, yielding the wide distribution of the lepton $\eta$.

Figure 6 (a, b) shows the NLO single (anti)top quark production cross section after applying the 'loose' cut set, for different $\eta_{j}^{\max }$ as a function of the jet $p_{T}$ threshold. It can be seen that an $\eta_{j}^{\max }$ of 2.5 as applied in our 'tight' cut set does not reduce the acceptance much compared to the $\eta_{j}^{\max }=5$ of the 'loose' cuts, but the reduction to very central regions of the detector greatly decreases the acceptance. The fraction of three-jet events is relatively large even up to high jet $p_{T}$ cuts, cf. Fig. 6 (c, d). Due to collinear enhancement from INIT corrections, the third jet has large pseudo-rapidity, so that the fraction of events with an additional jet greatly decreases when only considering central regions of the detector. In the following discussion of event distributions we will use the 'loose' cut set and $R_{\text {cut }}=0.4$. For the 'tight' cut set, the results are similar. 
(a)

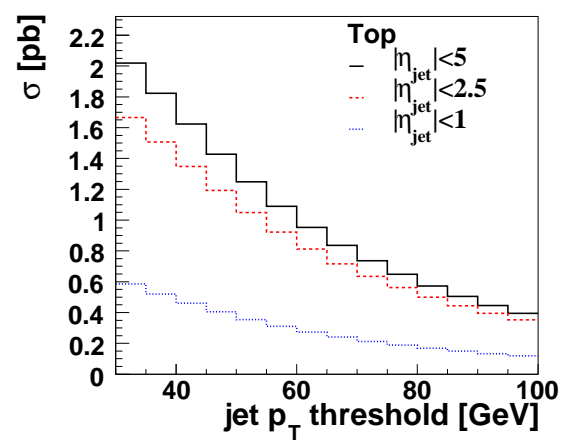

(c)

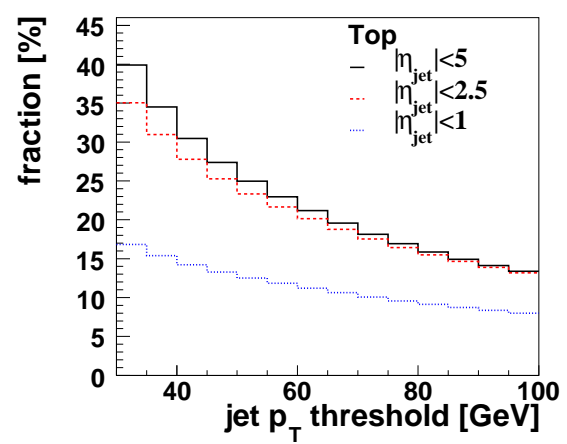

(b)

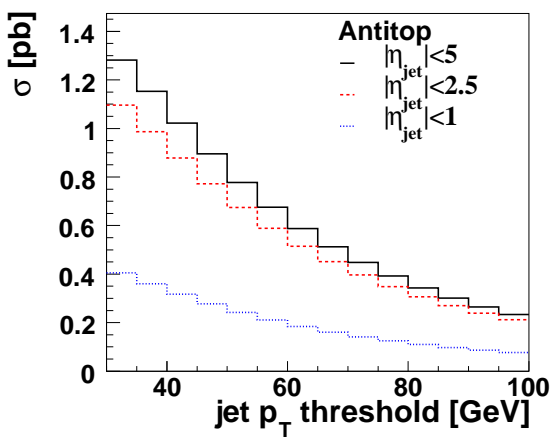

(d)

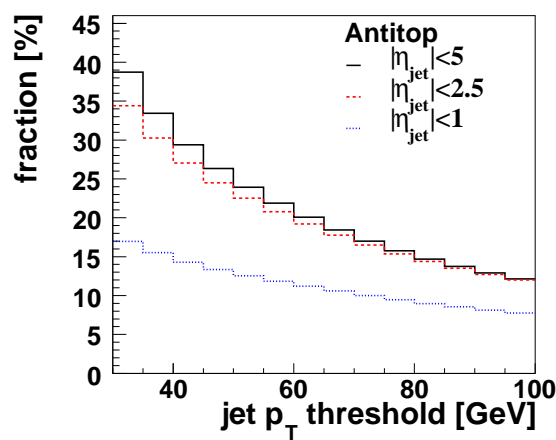

Figure 6: Single top and antitop quark production cross section and fraction of three-jet events at NLO for varying jet $p_{T}$ cuts, after applying the 'loose' cut set. (a, b) Total cross section for inclusive two-jet events as a function of the jet $p_{T}$ cut for three different jet $\eta$ cuts. (c, d) Fraction of exclusive three-jet events as a function of the jet $p_{T}$ cut for three different jet $\eta$ cuts.

\section{SINGLE TOP QUARK EVENT DISTRIBUTIONS}

In this section we discuss some of the kinematic properties of final state objects in schannel single top quark events. As described in Sec. III, the final state consists of one charged lepton, $\mathbb{E}_{T}$, two $b$ jets and possibly one additional jet due to NLO corrections. It is experimentally impossible to determine which of the $b$ jets is produced in association with the top quark and which comes from the top quark decay. With the third jet from NLO corrections, the situation becomes even more complicated, as the additional jet has to be correctly identified to come either from the production or decay of the top quark. A third jet coming from the SDEC correction should be included in the top quark reconstruction, while a third jet from INIT or FINAL corrections should not be. To find the best prescription for classifying the gluon/light quark jet correctly, we first examine various kinematical 
distributions of the final state particles. This includes a discussion of $b_{\text {fin }}$, the $\bar{b}(b)$ jet that is produced in association with the top (antitop) quark, and $b_{\text {dec }}$, which is the $b(\bar{b})$ jet from the top (antitop) quark decay. We then describe different prescriptions for the top quark reconstruction. After showing the superiority of the best-jet algorithm, we are able to reconstruct the top quark and discuss its kinematic properties. The kinematical distributions

of the final state particles are examined after applying the 'loose' set of selection cuts as discussed in Eq. (1).

\section{A. Final state object distributions}

\section{Leptons and missing transverse energy}

Figure 7 shows a comparison between the kinematical distributions of lepton and $E_{T}$ for both top and antitop quark production at LO and with $O\left(\alpha_{s}\right)$ corrections. As the distributions are for leptons and not quarks, the $O\left(\alpha_{s}\right)$ corrections do not change the general form of the $p_{T}$ distributions. The shapes of the distributions for the lepton $p_{T}$ look similar for top and antitop quarks. Furthermore, the $\mathbb{E}_{T}$ distributions peak at about $32 \mathrm{GeV}$ for both quark types. This is mainly because of a similar phase space in the transverse direction and similar spin correlations.

It can also be seen that the $E_{T}$ distribution peaks at a higher energy than the lepton $p_{T}$ distribution. This is due to spin correlations and the left-handedness of the weak interaction and illustrated in Fig. 8. The (anti)neutrino from the $W$ boson decays preferentially following the moving direction of the (anti)top quark and is therefore boosted in comparison to the lepton, which decays in the opposite direction when seen in the rest frame of the $W$ boson. The lepton $\eta$ distributions are the same for positive and negative values of $\eta$ as the $p p$ initial state of the LHC is parity symmetric. The NLO $\eta$ distribution for the positron from the top quark decay peaks at around $\eta=1.4$. This non-zero peak is due to LO and cannot be seen in the $O\left(\alpha_{s}\right)$ corrections. It originates from the longitudinal boost that the intermediate $W$ boson $\left(W_{\text {int }}\right)$ receives from the PDFs. For example, a single top quark is preferentially produced through collision of an up valence quark and a down antiquark from the quark sea. The valence quarks carry a large momentum fraction of the incoming proton, while the sea quarks carry a small fraction. As a result, the $(t \bar{b})$ system, which is equivalent to the $(u \bar{d})$ 
(a)

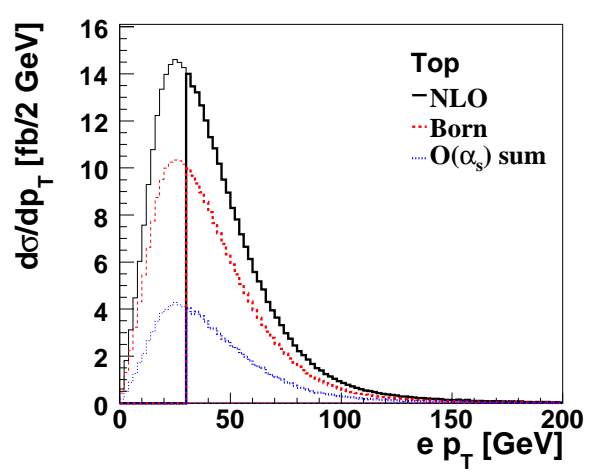

(c)

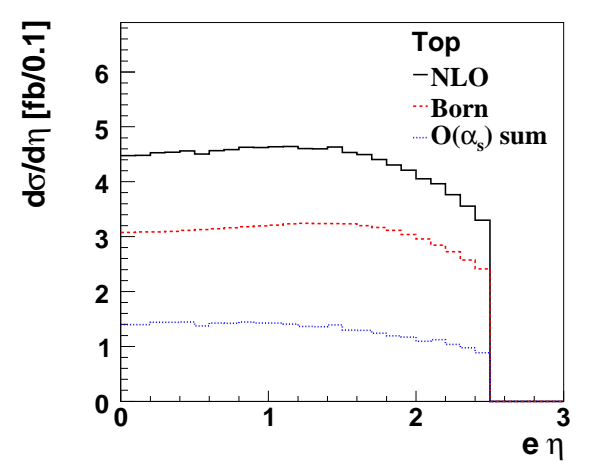

(e)

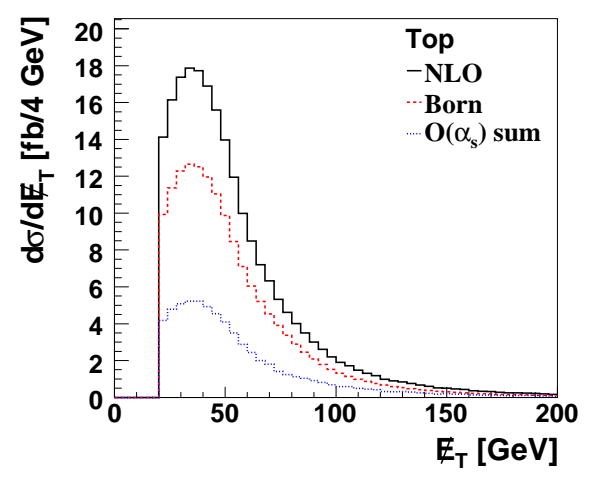

(b)

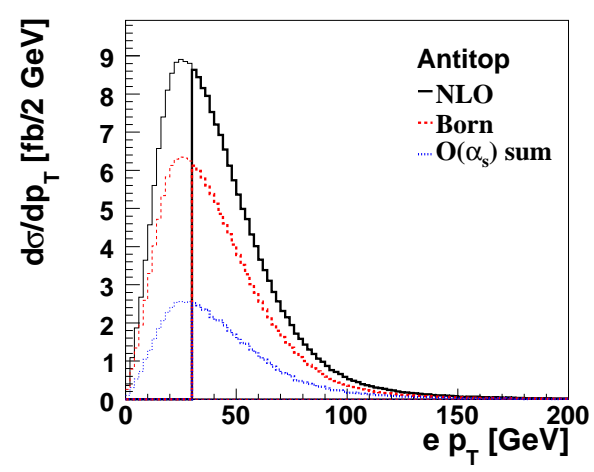

(d)

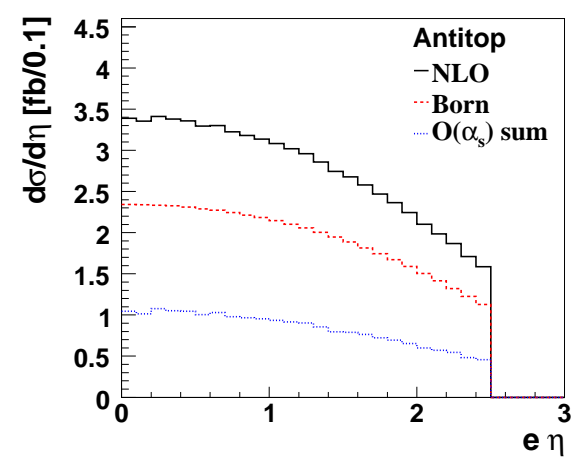

(f)

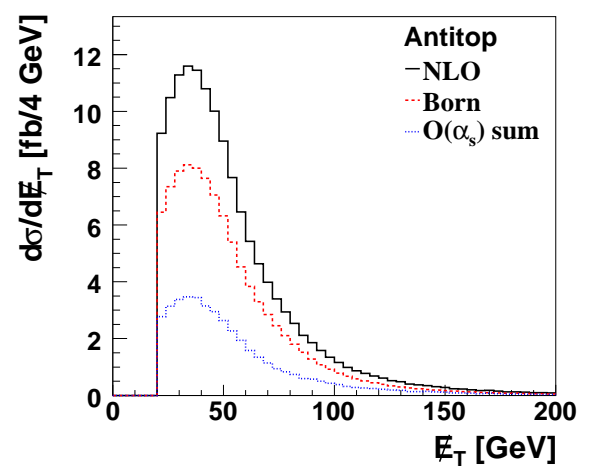

Figure 7: $p_{T}(\mathrm{a}, \mathrm{b})$ and $\eta(\mathrm{c}, \mathrm{d})$ for the lepton and $\mathbb{E}_{T}(\mathrm{e}, \mathrm{f})$ after selection cuts, comparing LO to $O\left(\alpha_{s}\right)$ corrections. (a, c, e) show top quark events, (b, d, f) antitop quark events. In (a, b) the distributions are also shown before the lepton $p_{T}$ cut.

system at LO, is naturally boosted along the direction of the incoming valence quark. This longitudinal boost is less strong for antitop quark production as in the antitop quark case a down valence quark collides with an up antiquark, with the down valence quark carrying a smaller momentum fraction than the up valence quark. At NLO, the reacting parton in the initial state could be a sea (anti-)quark or gluon, which results in a smaller difference in 
(a)

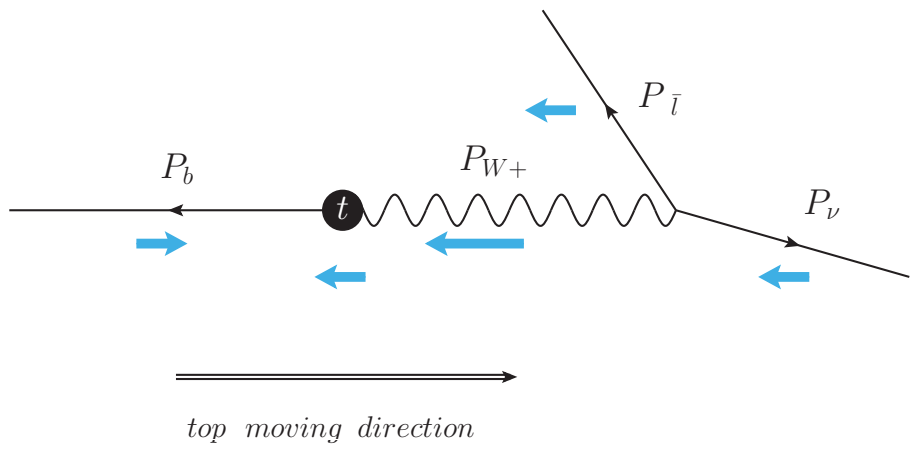

(b)

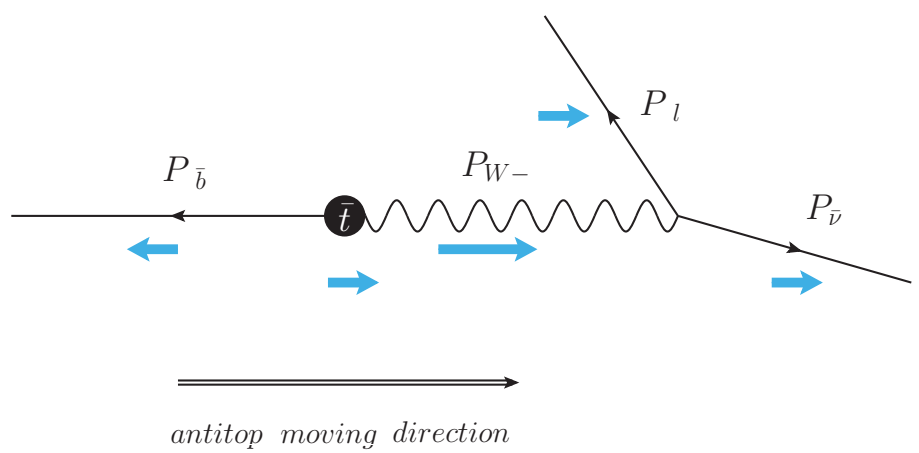

Figure 8: Spin correlations for top (a) and antitop (b) quark decay in the (anti)top quark rest frame. The thin lines describe momenta while bold lines indicate spins.

momenta between the colliding partons. Therefore, the lepton $\eta$ distributions of the $O\left(\alpha_{s}\right)$ corrections peak around zero.

\section{2. $b_{\text {fin }}$ and $b_{\text {dec }} j e t s$}

The comparison of the $p_{T}$ distributions of the $b_{\text {dec }}$ and $b_{\text {fin }}$ jets is shown in Fig. 9 for single top quark production. It is experimentally not possible to distinguish $b$ and $\bar{b}$ jets, but very instructive to consider their distributions individually. The $p_{T}$ Born distribution of the $b_{\text {dec }}$ jet peaks at roughly $1 / 3$ of $m_{t}$, as it is a top quark decay product, while that of the $b_{\text {fin }}$ jet peaks at lower $p_{T}$ and has a long tail to high $p_{T}$ values, because it is produced in association with the heavy top quark and has to balance the top quark $p_{T}$. The peak positions of both $b_{\text {fin }}$ and $b_{\text {dec }}$ jets are shifted to a slightly lower value by the QCD corrections. This is mainly due to the FINAL (SDEC) contribution because the emitted gluon tends to be collinear to the $b_{f i n}\left(b_{d e c}\right)$ jet in the FINAL (SDEC) correction. Furthermore, the NLO 
(a)

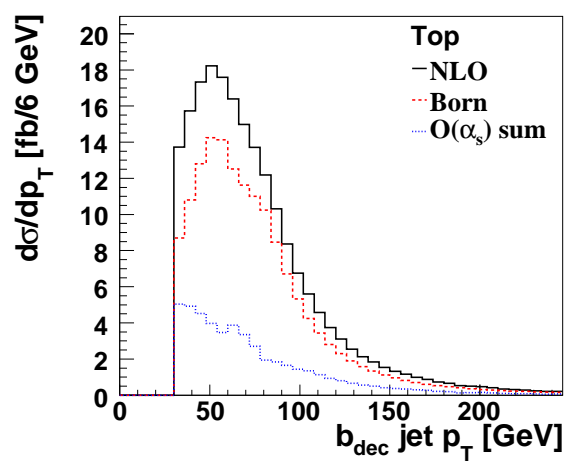

(c)

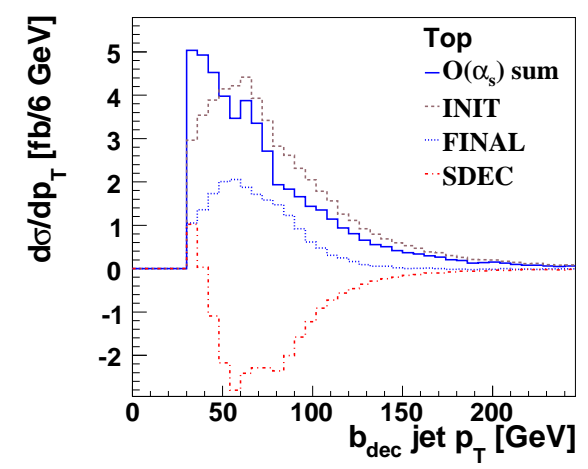

(b)

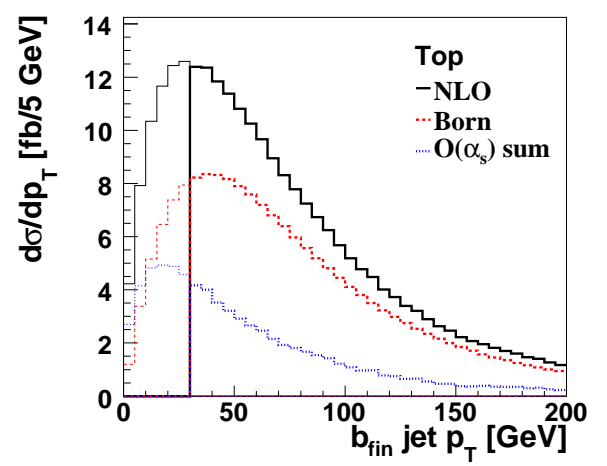

(d)

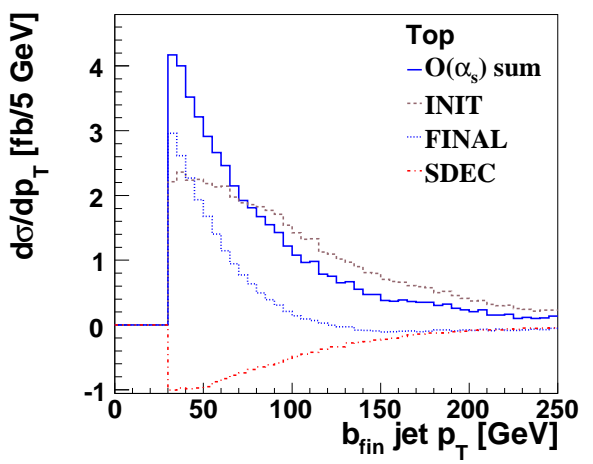

Figure 9: $p_{T}$ distributions for the $b_{d e c}$ jet from the top quark decay $(\mathrm{a}, \mathrm{c})$ and the $b_{\text {fin }}$ jet $(\mathrm{b}, \mathrm{d})$ after selection cuts [(b) is also shown before the $b_{\text {fin }}$ jet $p_{T}$ cut]. (a, b) show NLO, Born and sum of $O\left(\alpha_{s}\right)$ contributions, (c, d) individual $O\left(\alpha_{s}\right)$ contributions.

distribution is broadened due to the INIT contribution which adds additional $p_{T}$ to the event by emitting a third jet in the initial state. Note that among the three $O\left(\alpha_{s}\right)$ corrections the INIT contribution dominates as it receives both soft and collinear enhancements at NLO. The soft gluon contributions in the FINAL and SDEC contributions are suppressed in comparison to the INIT correction, due to the large top quark mass. The single antitop quark production exhibits very similar distributions and is not shown here.

Figure 10 shows the $\eta$ distributions of the $b_{\text {fin }}$ and $b_{\text {dec }}$ jet for both single top and antitop quark production. For top quark production the Born distribution of the $b_{\text {dec }}$ jet $\eta$ is much broader than the Born distribution of the $b_{\text {fin }}$ jet $\eta$. On the other hand, for antitop production the $b_{\text {fin }}$ jet exhibits a wider $\eta$ distribution than the $b_{\text {dec }}$ jet. This is due to spin correlations and the boost of $W_{\text {int }}$ along the beam line. Figure 11 shows single top and antitop quark production in the c.m. frame of $W_{i n t}$. Due to spin conservation, for top quark 
(a)

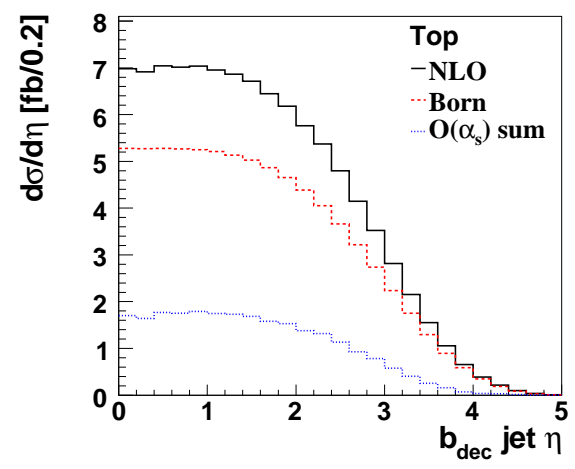

(c)

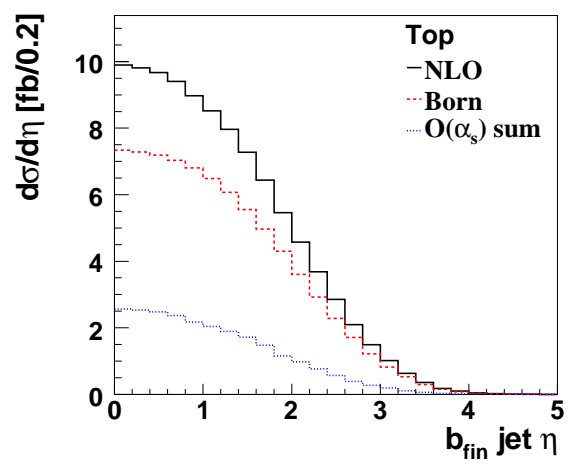

(b)

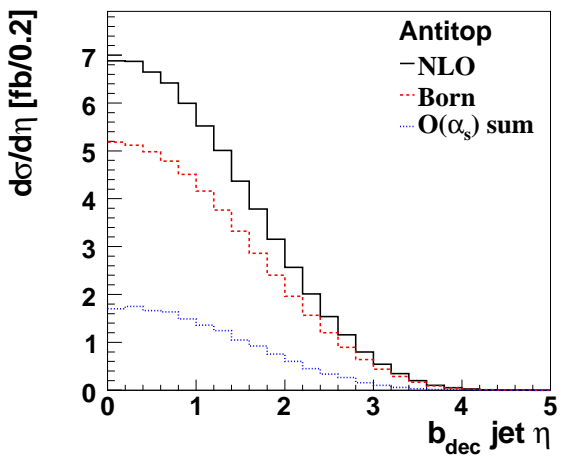

(d)

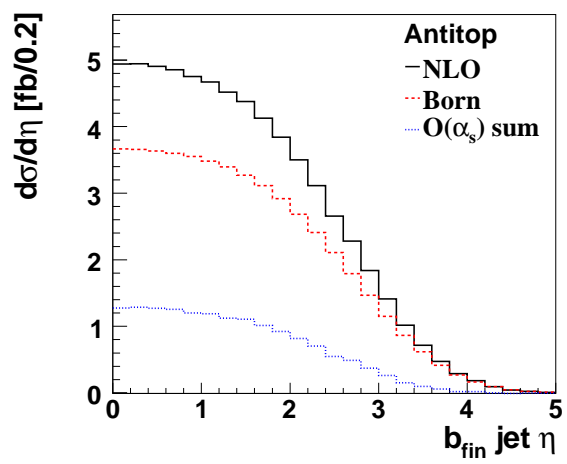

Figure 10: $\eta$ distributions of the $b_{\text {dec }}$ jet from the top/antitop quark decay $(\mathrm{a} / \mathrm{b})$ and the $b_{\text {fin }}$ jet produced in association with the top/antitop quark (c/d) after applying the 'loose' cut set.

(a)

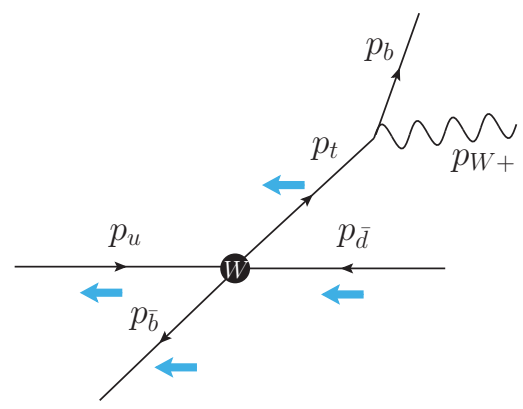

$W_{\text {int }}$ moving direction (b)

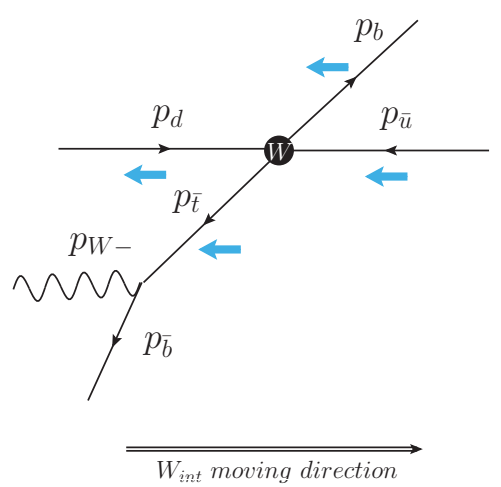

Figure 11: Pictorial illustration of spin correlations and boost effects in top (a) and antitop (b) quark production in the c.m. frame of $W_{\text {int }}$. The thin lines denote particle momenta while the bold lines label spin directions. 
(a)

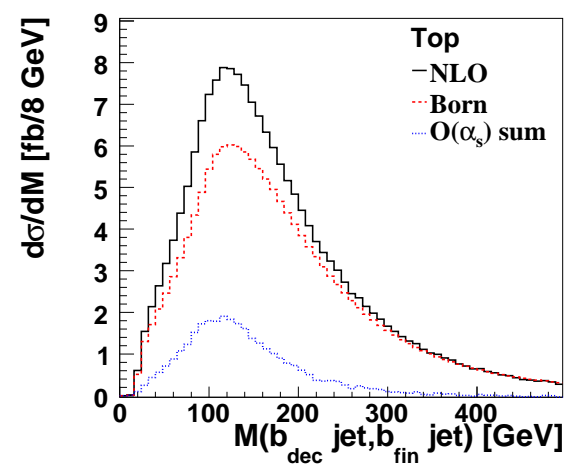

(b)

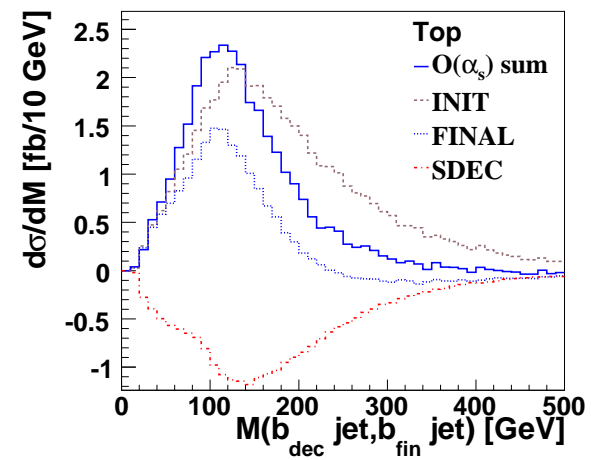

Figure 12: Invariant mass of the $\left(b_{\text {dec }}\right.$ jet, $b_{\text {fin }}$ jet) system after selection cuts, comparing Born level to $O\left(\alpha_{s}\right)$ corrections.

production, in the c.m. frame of $W_{\text {int }}$, the top quark mainly follows the direction of motion of the incoming up quark, while the $b_{\text {fin }}$ jet $[\bar{b}$ quark in Fig. 11 (a)] follows the direction of motion of the incoming antiquark. Furthermore, in the top quark production process, $\bar{b}$ is right-handed and $b$ is left-handed due to the left-handed charged current interaction in the SM. Since the valence quarks carry a larger fraction of the proton momentum, $W_{i n t}$ is boosted along the direction of motion of the up quark. The top quark and its decay product, the $b_{\text {dec }}$ jet, go in the same direction, and therefore have a larger longitudinal momentum and higher $\eta$ than the $b_{\text {fin }}$ jet, which, in the c.m. frame of $W_{i n t}$, follows the opposite direction and therefore receives a smaller longitudinal boost. For the antitop quark production it is the $b_{\text {fin }}$ jet [ $b$ quark in Fig. 11 (b)] and not the antitop quark that, in the c.m. frame of $W_{\text {int }}$, follows the direction of motion of the incoming quark. Therefore the $\eta$ distribution of the $b_{\text {fin }}$ jet is wider than the same distribution of the $b_{\text {dec }}$ jet from the antitop quark decay. As mentioned in Sec. IV A 1, the boost is smaller for antitop quark production, so the effect on the $\eta$ width is less strong.

The $O\left(\alpha_{s}\right)$ corrections shift the $b_{\text {fin }}$ jet produced in association with the top quark to more central rapidities. This effect is not very strong and mainly due to initial state corrections, which add to the $p_{T}$ of the event while reducing the boost along the beam line direction. The shape of the $\eta$ distribution of the $b_{\text {dec }}$ jet remains practically unchanged by the $O\left(\alpha_{s}\right)$ corrections.

Single top quark production is an irreducible background to the Higgs boson search, e.g. $W^{ \pm} H$ associated production with the subsequent Higgs boson decay $H \rightarrow b \bar{b}$. Even though 
$W^{ \pm} H$ production is not the largest production channel at the LHC, one has to combine its contribution with other channels in order to reach $5 \sigma$ statistics significance. Thus it is crucial to have a good understanding of the SM backgrounds. Since a light Higgs boson predominantly decays into a $b \bar{b}$ pair, we examine the impact of $O\left(\alpha_{s}\right)$ corrections on the invariant mass distribution of the $b \bar{b}$ pair in single top quark production. Figure 12 plots the invariant mass distribution of the $\left(b_{\text {dec }}\right.$ jet, $b_{f i n}$ jet) system. It can be seen that the FINAL correction shifts the peak of the invariant mass to slightly lower values. This is the case if a third jet is produced in addition to the $b_{d e c}$ jet and the $b_{f i n}$ jet. The INIT corrections tend to have peaks at higher invariant mass, due to the additional $p_{T}$ they provide. The drop-off at higher mass values is faster at NLO level. The invariant mass distribution of the two $b$ jets for antitop quark events looks very similar in spite of the differences in initial state PDFs.

\section{Invariant mass $M\left(b_{\text {dec }}\right.$ jet, lepton $)$}

The top quark decays into a $b_{d e c}$ jet and a $W$ boson, which itself can decay leptonically. The invariant mass distribution of the $\left(b_{\text {dec }}\right.$ jet, lepton) system is characteristic of the decay

of the SM top quark and sensitive to the $W$-t-b coupling (or the $W$ boson helicity) [61, 62],

$$
m_{b_{j e t} \ell}^{2} \approx \frac{1}{2}\left(m_{t}^{2}-m_{W}^{2}\right)\left(1-\cos \theta_{\ell}^{\star}\right)
$$

where $\theta_{\ell}^{\star}$ is the polar angle of the charged lepton in the rest frame of the $W$ boson which is defined in the rest frame of the top quark. Figure 13 shows the drop-off behavior of the invariant mass distribution at $\sqrt{m_{t}^{2}-m_{W}^{2}} \approx 155 \mathrm{GeV}$, due to the kinematics of the event. It also shows that the SDEC correction shifts the invariant mass peak to lower values, mainly because it weakens the spin correlations.

\section{4. $H_{T}$ distributions}

In order to distinguish the s-channel single top quark mode from dominant backgrounds, it is important to know how the total transverse energy $H_{T}$ changes with NLO corrections. In Fig. 14 we look at the effect that $O\left(\alpha_{s}\right)$ corrections have on the total transverse energy 
(a)

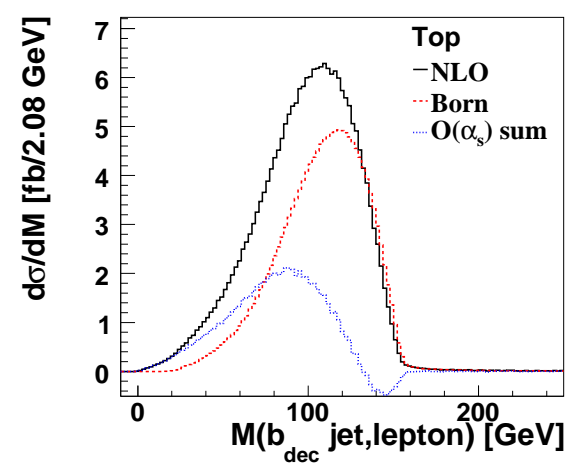

(b)

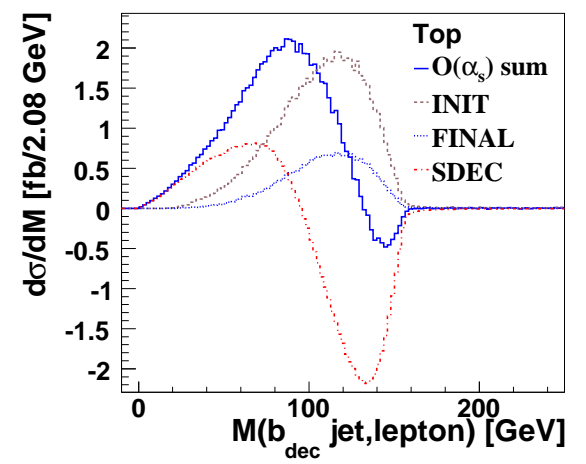

Figure 13: Invariant mass of the ( $b_{\text {dec }}$ jet, lepton) system after selection cuts, comparing Born level to $O\left(\alpha_{s}\right)$ corrections.

(a)

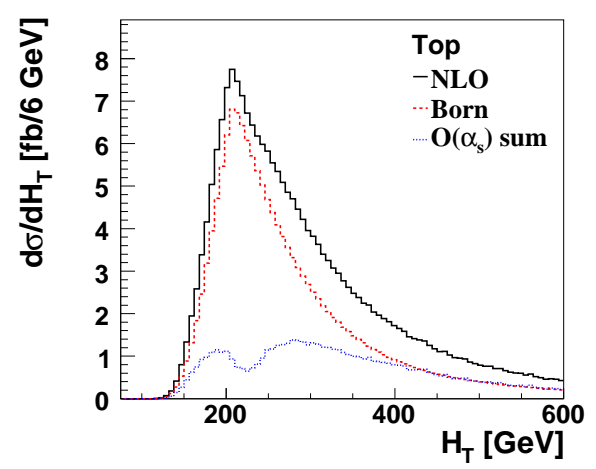

(b)

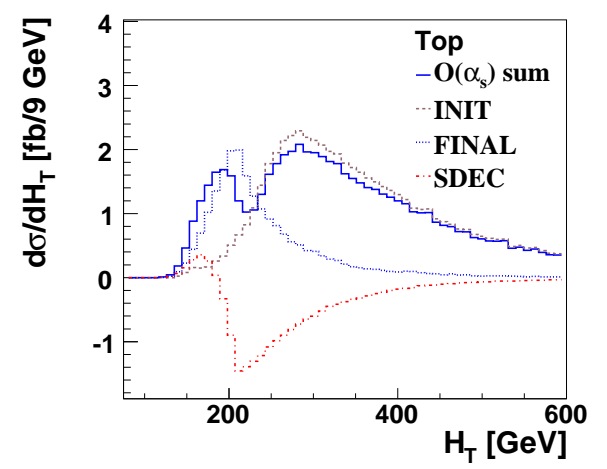

Figure 14: Comparison of the total transverse energy of single top quark events after selection cuts between LO and NLO (a) and between the different $O\left(\alpha_{s}\right)$ corrections (b).

$H_{T}$ of a single top quark event. $H_{T}$ is defined as

$$
H_{T}=p_{T}^{\text {lepton }}+E_{T}+\sum_{j e t s} p_{T}^{j e t}
$$

Clearly, the SDEC and FINAL $O\left(\alpha_{s}\right)$ contributions shift the total transverse energy down, while the INIT contribution shifts it up. This again is due to the additional transverse energy that a third jet in the initial state adds to the event. The shift to higher transverse energies is significant for single top quark measurements at the LHC, as $t \bar{t}$ events are generally a larger background for higher $H_{T}$ values. 


\section{B. Event reconstruction}

One of the main reasons for studying single top quark events is to find out more about the properties of the top quark and its couplings. Furthermore, as single top quark production is a weak interaction process, we expect a number of correlations between the particles. It is therefore of interest to reconstruct the complete event, including final state jets and intermediate particles.

As explained in Refs. [40, 63], it is possible to reconstruct the $W$ boson from the observed charged lepton and $\mathbb{E}_{T}$, where the unknown longitudinal momentum of the neutrino $p_{z}^{\nu}$ is substituted by the restriction that the invariant mass of the (charged lepton, neutrino) system has to equal the mass of the $W$ boson. Of the two possible solutions we pick the one with the smaller $\left|p_{z}^{\nu}\right|$. For the top quark reconstruction, we must then combine the reconstructed $W$ boson with the $b_{\text {dec }}$ jet from the top quark decay. This means we have to identify the correct jet as the $b_{\text {dec }}$ jet. There are several different methods to select this jet. As both the $b_{d e c}$ jet and the $b_{\text {fin }}$ jet have high $p_{T}$ and are possibly b-tagged, neither the method of choosing the leading jet (jet with the highest $p_{T}$ ) nor the second jet (jet with the second highest $p_{T}$ ) nor the b-tagged jet is very reliable in identifying the right jet.

A more effective algorithm is the so-called best-jet algorithm as explained in Ref. [64] and more specifically for our case in Ref. [40]. Here, the $W j$ or the $W j j$ (only possible in cases where there is a third jet) combination, that gives an invariant mass closest to the input $m_{t}$, is chosen as the reconstructed top quark and the jet (or two-jet system), that is thus identified, is called best-jet. Of the two possible other jets, the one with larger $p_{T}$ is the so-called non-best-jet.

Figure 15 shows the efficiencies of the best-jet, the leading jet (jet 1) and the second jet (jet 2) algorithms. The leading jet has an overall efficiency of 39\%. The second jet mainly corresponds to the $b_{d e c}$ jet for very high $p_{T}$ and has an overall efficiency of about $49 \%$, while the best-jet algorithm has a high efficiency for all momenta and identifies the $b_{\text {dec }}$ jet correctly in $93 \%$ of all events. Its effectiveness is mostly limited by the efficiency of the $W$ boson identification; with a falsely reconstructed $W$ boson, the identification of the $b_{\text {dec }}$ jet by the best-jet algorithm becomes a random pick.

The invariant mass of the $W j$ combination is shown in Fig. 16, comparing the reconstruction using the best-jet, the leading jet and the second jet algorithm to the reconstruction 


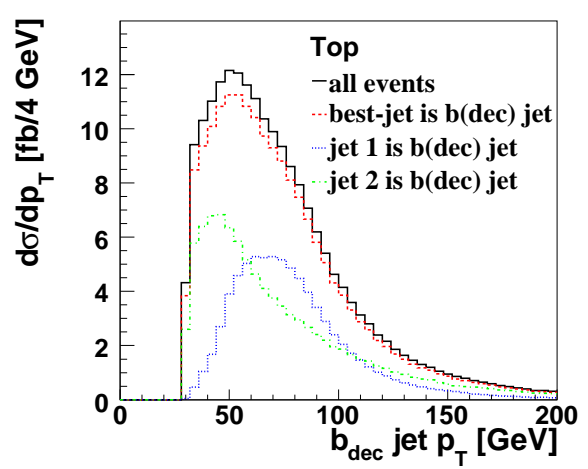

Figure 15: $p_{T}$ distribution of the $b_{d e c}$ jet from the top quark decay, for all events that pass the 'loose' set of cuts (solid), only for those events in which the $b_{d e c}$ jet is the best-jet (dashed), only for events in which the $b_{d e c}$ jet is also the leading jet (dotted) and only for events in which the $b_{\text {dec }}$ jet is the second jet (dash dotted).

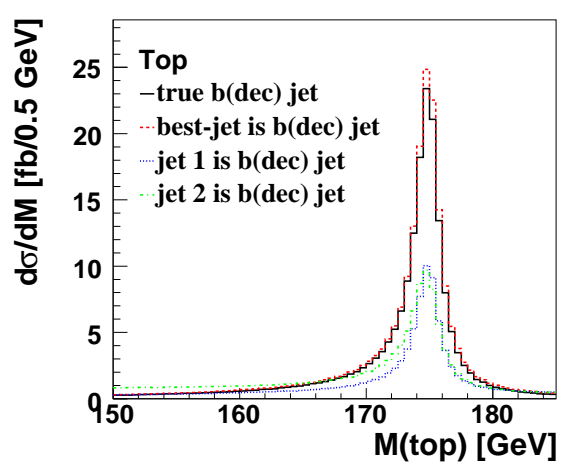

Figure 16: Invariant mass of the $W j$ combination after selection cuts, where the jet $j$ is the real $b_{\text {dec }}$ jet (solid), the best-jet (dashed), the leading jet (dotted) and the second jet (dash dotted).

using the real $b_{d e c}$ jet. For the identification of the real $b_{d e c}$ jet, parton level information is used and a possible third jet from the top quark decay is included. For all four curves, the $W$ boson is reconstructed from the final state lepton and $E_{T}$, so that all differences in shape and height are due to the method of identifying the $b_{\text {dec }}$ jet. As expected, the distribution using the best-jet fits the distribution with the true $b_{d e c}$ jet information much better than the distribution using the leading jet or the second jet. It is important to notice though, that this is due to two competing effects: (i) The best-jet does not identify the correct $b_{\text {dec }}$ jet in $7 \%$ of all events, which reduces the height of the distribution in Fig. 15: (ii) Due to the requirement to be as close as possible to the input $m_{t}$, mis-reconstructed events are shifted closer to $175 \mathrm{GeV}$, increasing the height of the peak. The second effect is larger than the 
(a)

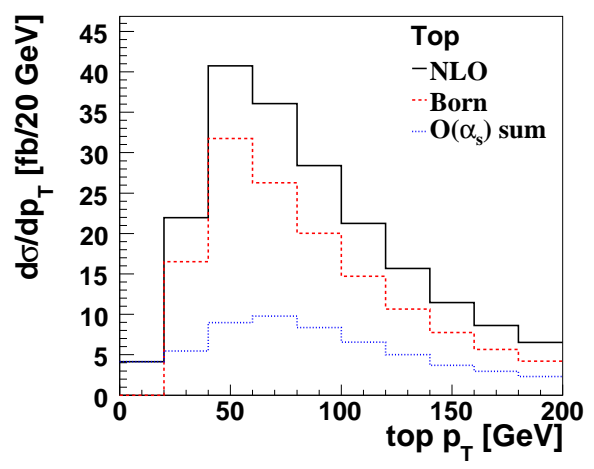

(c)

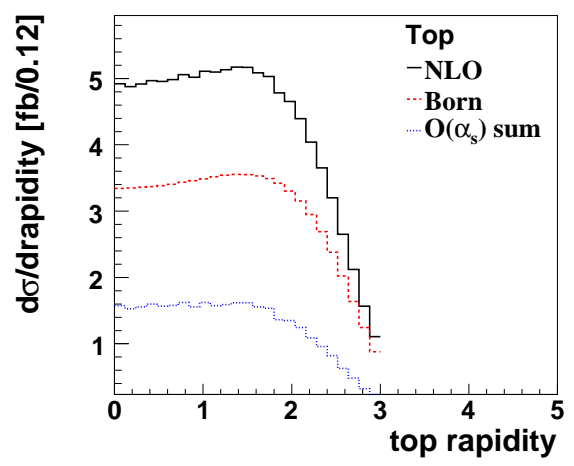

(b)

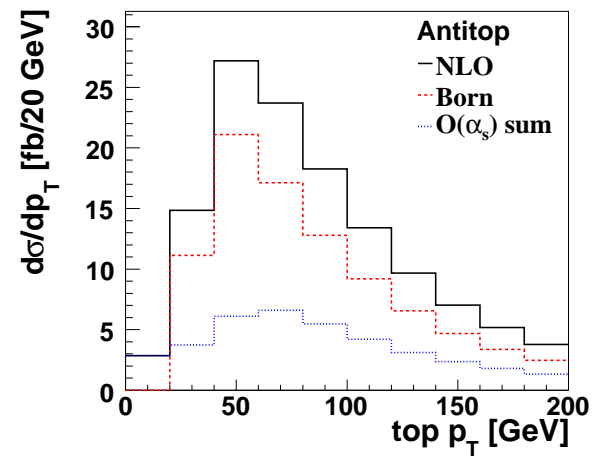

(d)

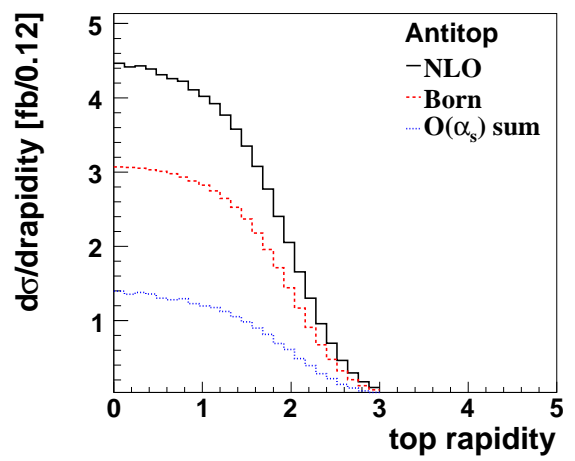

Figure 17: Distributions of $p_{T}(\mathrm{a}, \mathrm{b})$ and rapidity $(\mathrm{c}, \mathrm{d})$ of the top quark $(\mathrm{a}, \mathrm{c})$ and of the antitop quark (b, d), both reconstructed with the best-jet algorithm and after applying the 'loose' set of selection cuts.

first, so that the peak reconstructed with the best-jet algorithm is actually higher than the peak reconstructed using the real $b_{\text {dec }}$ jet.

We reconstruct the top quark using the best-jet algorithm and are now able to study some of its kinematic properties. In Fig. 17, the $p_{T}$ and rapidity distributions are shown for both top and antitop quark, reconstructed with the best-jet algorithm and after applying the 'loose' set of selection cuts. The shapes of the $p_{T}$ distributions look similar, but the rapidity distribution is wider for the top quark than for the antitop quark. This is a reflection of the boosted kinematics of $W_{\text {int }}$ and the (anti)top quark itself (cf. Fig. 11). The NLO corrections do not significantly change the the shape of the distributions.

We reconstruct the virtual $W$ boson $W_{\text {int }}$ in single top quark production by combining the reconstructed top quark with the non-best-jet. This method is exact if best-jet and non-best-jet are identified correctly and the event does not contain a third jet from $O\left(\alpha_{s}\right)$ 
(a)

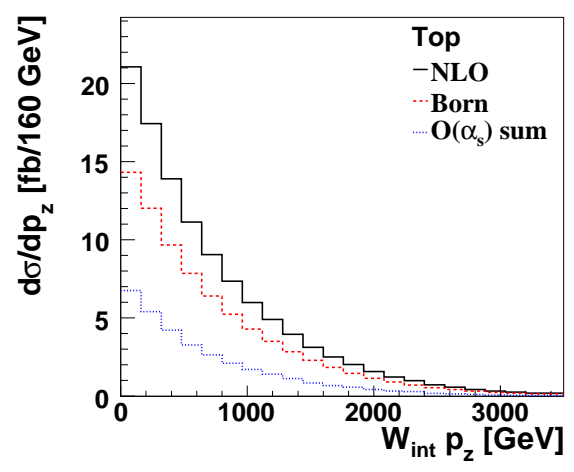

(c)

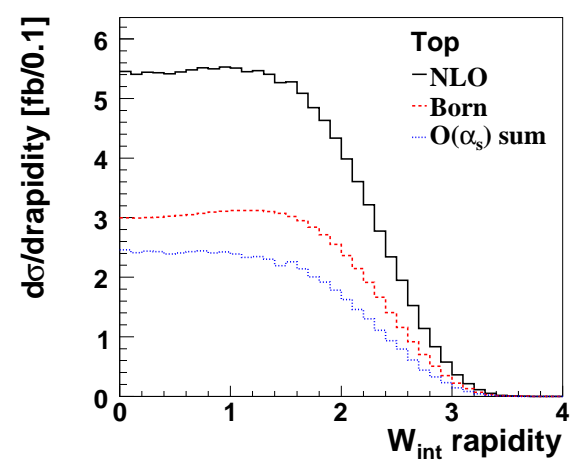

(e)

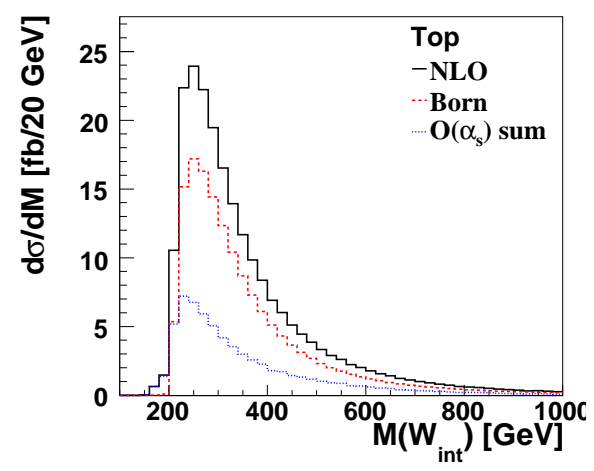

(b)

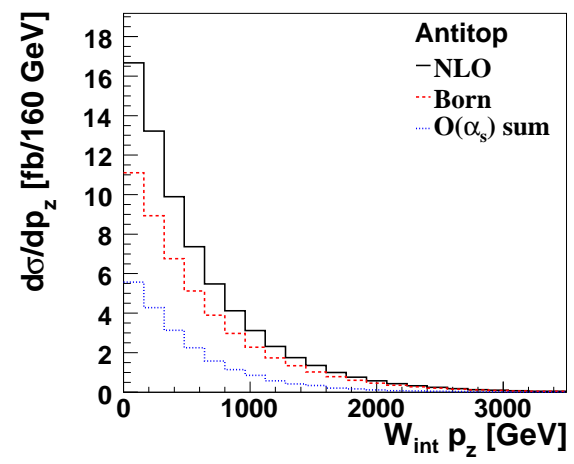

(d)

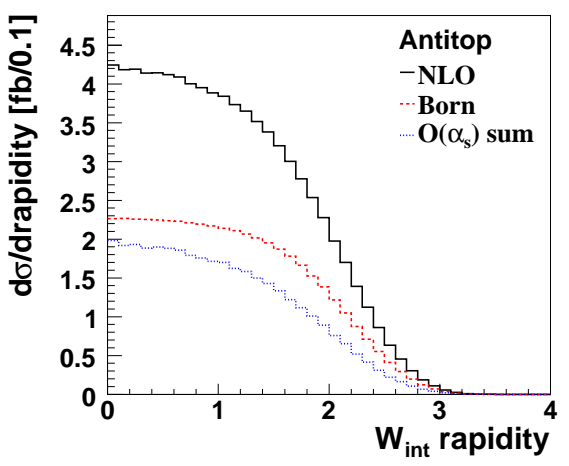

(f)

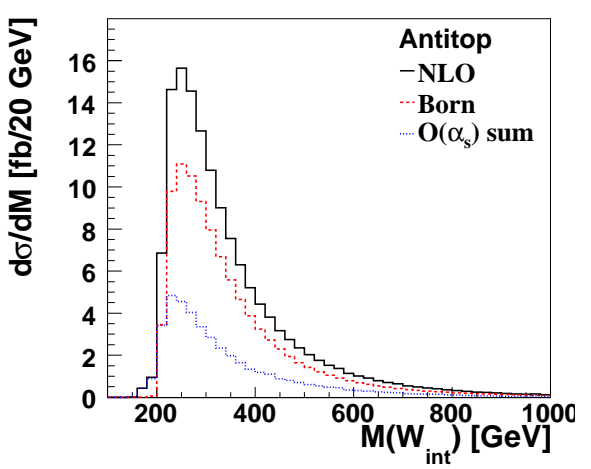

Figure 18: $p_{z}(\mathrm{a}, \mathrm{b})$, rapidity $(\mathrm{c}, \mathrm{d})$ and invariant mass $(\mathrm{e}, \mathrm{f})$ distributions of the intermediate $W$ boson after selection cuts. (a, c, e) show top quark production, (b, d, f) antitop quark production. The rapidity distribution only contains events with $p_{T} \neq 0$.

FINAL corrections. As discussed in Sec. IV A 1, at LO $W_{\text {int }}$ receives a longitudinal boost, which is stronger for top than for antitop quark production. Figure 18 shows that the LO $p_{z}$ and rapidity distributions are wider for $W_{\text {int }}$ in top than in antitop quark production. Furthermore, the $O\left(\alpha_{s}\right)$ correction to the rapidity distribution of $W_{\text {int }}$ is narrower than the LO contribution. This is because at NLO the incoming partons could also be sea quarks or 
(a)

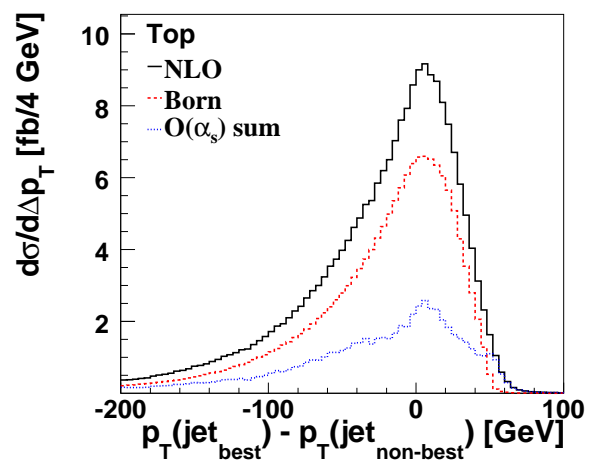

(b)

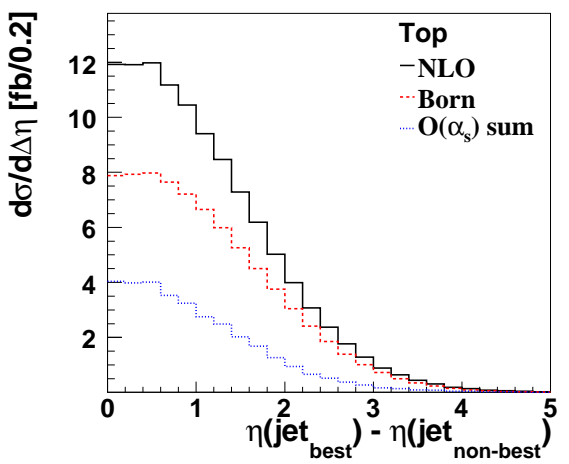

Figure 19: Difference in $p_{T}$ (a) and $\eta$ (b) between the best-jet and the non-best-jet, after applying the 'loose' set of kinematic cuts.

gluons which results in a less boosted $W_{\text {int }}$ system.

In order to search for new physics in the form of a $W^{\prime}$ boson, it is important to know the invariant mass distribution of the intermediate SM $W$ boson. $W^{\prime}$ boson searches in the single top quark final state have been performed at the Tevatron and have set lower $W^{\prime}$ boson mass limits in the range $750 \mathrm{GeV}$ to $800 \mathrm{GeV}$ [18 20]. The LHC with its higher $E_{c . m}$. will allow to probe up to even larger boson masses. The invariant mass distribution of $W_{\text {int }}$ is shown in Fig. 18 for SM single top and single antitop quark production. The $O\left(\alpha_{s}\right)$ corrections, more specifically the FINAL contribution, shift the invariant mass peak to lower energies, as in our reconstruction of $W_{\text {int }}$ we do not include a possible third jet in the final state.

\section{Kinematical and spin correlations}

\section{Correlations between the $b$ jets}

Having reconstructed and identified the $W$ boson, the $b_{\text {dec }}$ jet and the $b_{\text {fin }}$ jet, it is now possible to study correlations between objects in single top quark events. Figure 19 shows that the $b_{\text {dec }}$ jet (best-jet) and the $b_{\text {fin }}$ jet (non-best-jet) in single top quark events are highly correlated. The correlations are modified by the $O\left(\alpha_{s}\right)$ corrections. The FINAL corrections shift the $p_{T}$ difference between best-jet and non-best-jet to more positive values, as their tendency to be collinear to the boost direction lowers the $p_{T}$ of the $b_{f i n}$ jet. For the same reason, the $\eta$ correlation is shifted to slightly more central values. 


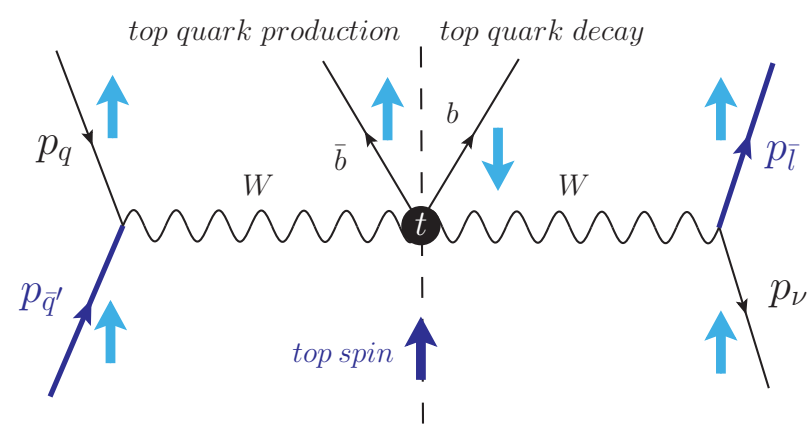

Figure 20: S-channel single top quark production from the perspective of a top quark at rest. Thin arrows denote directions of momentum while bold arrows indicate spin directions.

\section{Top quark polarization}

The SM predicts that single top quarks are highly polarized. Verifying this prediction can be used as a test of the SM electroweak symmetry breaking mechanism and a check for new physics [8, 23, 24, 65]. It is in principle possible to measure the polarization of the single top quark, by making use of the fact that the charged lepton from the top quark decay is maximally correlated with the top quark spin [66, 67], as illustrated in Fig. 8, In the following we therefore plot the angle between the charged lepton and a reference axis in the rest frame of the top quark. Two different choices for this reference frame have been used in the past: the helicity basis and the so-called optimal basis [8, 66 68]. In the helicity basis, the top quark spin is measured along the top quark direction of motion in the center of mass (c.m.) frame of the system. In the optimal basis, the top (antitop) quark spin is measured along the direction of the incoming antiquark (quark) in the c.m. frame of the top (antitop) quark. This reference frame is called the optimal basis, as the top (antitop) quark produced in s-channel single top quark processes is almost 100\% polarized along (against) the direction of the incoming antiquark (quark). Figure 20 shows s-channel single top quark production from the perspective of a top quark at rest. It demonstrates how the optimal basis makes use of the momentum - spin correlations that are due to spin conservation and left-handedness of the weak interaction.

While at the Tevatron the antiquark comes predominantly from the antiproton, the LHC is a $p p$ collider and there is no preferred direction for the antiquark. We can however make use of the fact that the incoming quark is more likely to be a valence quark, and has a larger 
longitudinal momentum than the antiquark which comes always from the quark sea of the proton. In most cases the direction of the longitudinal boost of $W_{i n t}$ therefore indicates the direction of the incoming quark. (This becomes a better approximation when the magnitude of the longitudinal momentum $\left|p_{z}\right|$ of $W_{\text {int }}$ is large.) We choose the reference axis according to the sign of $p_{z}\left(W_{i n t}\right)$. After discussing the helicity basis, we show how it is possible to enhance the spin correlations for the optimal basis further by requiring a minimum $p_{z}\left(W_{\text {int }}\right)$.

For the helicity basis, the c.m. frame of the system has to be reconstructed in order to define the top quark momentum. As discussed in Ref. [41], this is more complicated at NLO than at Born level, because of additional jets. Ref. [41] investigates two options for reconstructing the c.m. frame: the $t b_{\text {fin }}(j)$-frame, which is the c.m. frame of the incoming partons and the rest frame of all the final state objects (reconstructed top quark and all other jets), and the $t b_{\text {fin }}$-frame, which is the c.m. frame of the top quark and the non-best-jet. The $t b_{\text {fin }}$-frame differs from the $t b_{f i n}(j)$-frame only in exclusive three-jet events. As shown in Table $\mathrm{V}$ and discussed below, the degree of polarization is larger in the $t b_{f i n}$-frame, so we only show distributions for the top quark polarization in this frame.

In the helicity basis, we examine the polarization of the top quark by studying the angular distribution $\cos \theta_{\text {hel }}$ of the lepton relative to the moving direction of the top quark, both in the c.m. frame of the system,

$$
\cos \theta_{h e l}=\frac{\vec{p}_{t} \cdot \vec{p}_{\ell}^{*}}{\left|\vec{p}_{t}\right|\left|\vec{p}_{\ell}^{*}\right|},
$$

where $\vec{p}_{t}$ is the top quark three-momentum defined in the $t b_{f i n^{-}}$or the $t b_{f i n}(j)$-frame, and $\vec{p}_{\ell}^{*}$ is the charged lepton three-momentum, after boosting it first into the c.m. frame of the system and then into the top quark rest frame. For a left-handed top quark, the angular correlation of the lepton $\ell^{+}$is given by $\left(1-\cos \theta_{\text {hel }}\right) / 2$, and for a right-handed top quark, it is $\left(1+\cos \theta_{\text {hel }}\right) / 2$. For a right-handed antitop quark, the angular correlation of the lepton $\ell^{-}$is given by $\left(1-\cos \theta_{\text {hel }}\right) / 2$, and for a left-handed antitop quark, it is $\left(1+\cos \theta_{\text {hel }}\right) / 2$.

Figure 21 shows that this linear relationship for $\cos \theta_{\text {hel }}$ indeed describes s-channel single top quark events well at parton level. It also shows that the top quark is not completely polarized in the helicity basis, and that this polarization is weakened further at NLO. After event reconstruction, the drop-off close to $\cos \theta_{h e l}=-1$ is due to jet-lepton separation cuts.

The corresponding distributions for the optimal basis can be seen in Fig. 22. Here, the 
(a)

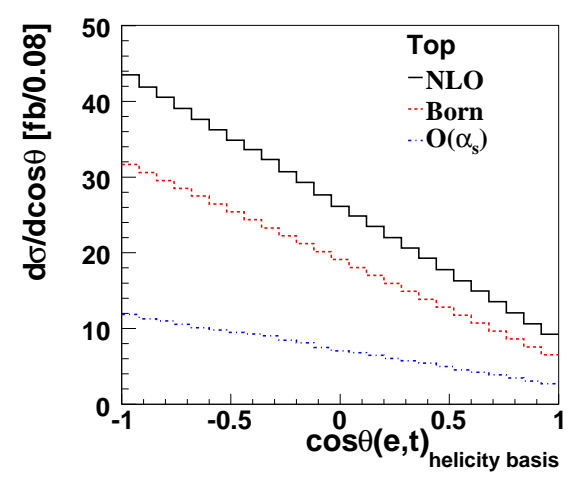

(c)

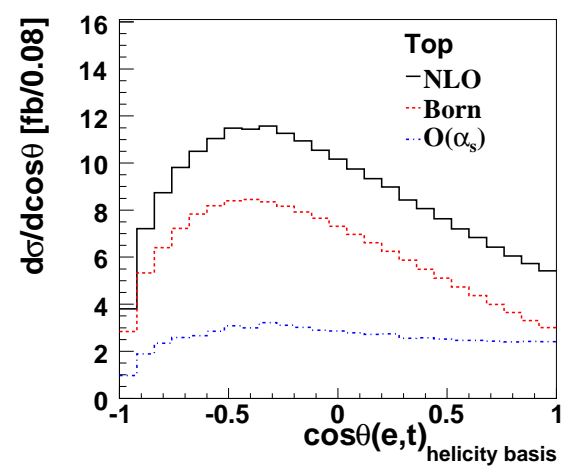

(b)

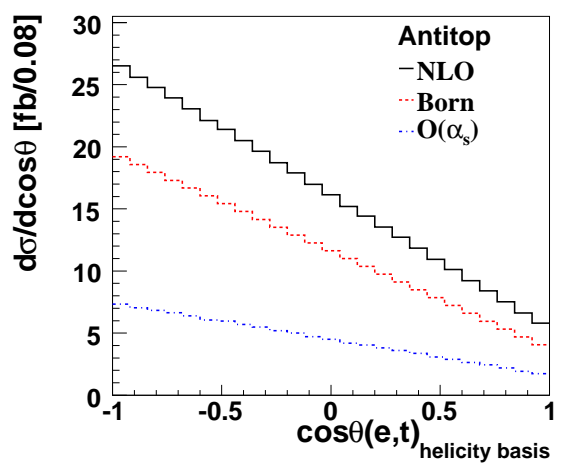

(d)

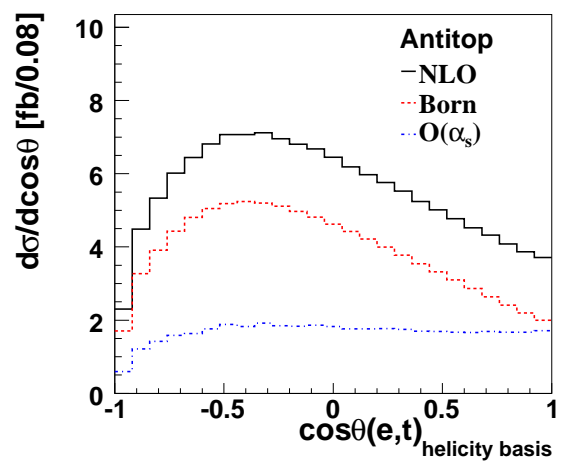

Figure 21: Top quark polarization in the helicity basis at parton level $(\mathrm{a}, \mathrm{b})$ and after event reconstruction and applying the 'loose' set of selection cuts (c, d), for top quark production (a, c) and antitop quark production $(\mathrm{b}, \mathrm{d})$. The $t b_{f i n}$-frame is chosen as the c.m. frame.

relevant angular correlation is

$$
\cos \theta_{\text {opt }}=\frac{\vec{p}_{p_{1}} \cdot \vec{p}_{\ell}^{*}}{\left|\vec{p}_{p_{1}}\right|\left|\vec{p}_{\ell}^{*}\right|}
$$

where $\vec{p}_{p_{1}}$ is the three-momentum in the top quark rest frame of the proton which travels (in the case of top quark production) antiparallel to the longitudinal boost that $W_{\text {int }}$ receives, e.g. in the $-\overrightarrow{p_{z}}\left(W_{\text {int }}\right)$ direction. In the case of antitop quark production the chosen proton travels parallel to the longitudinal boost of $W_{i n t}$, e.g. in the $\overrightarrow{p_{z}}\left(W_{i n t}\right)$ direction. $\overrightarrow{p_{\ell}^{*}}$ is the three-momentum of the lepton in the top quark rest frame. For a(n) (anti)top quark polarized along (against) the moving direction of the chosen proton, the angular distribution of the lepton $\ell^{+}\left(\ell^{-}\right)$is $\left(1+\cos \theta_{\text {opt }}\right) / 2$, while for $\mathrm{a}(\mathrm{n})$ (anti)top quark polarized against (along) the moving direction of the chosen proton, it is $\left(1-\cos \theta_{\text {opt }}\right) / 2$. Figure 22 shows that there is indeed a linear relationship for $\cos \theta_{\text {opt }}$ at parton level, but that the top quark is not completely polarized in this basis. After reconstruction and applying the 'loose' set 
(a)

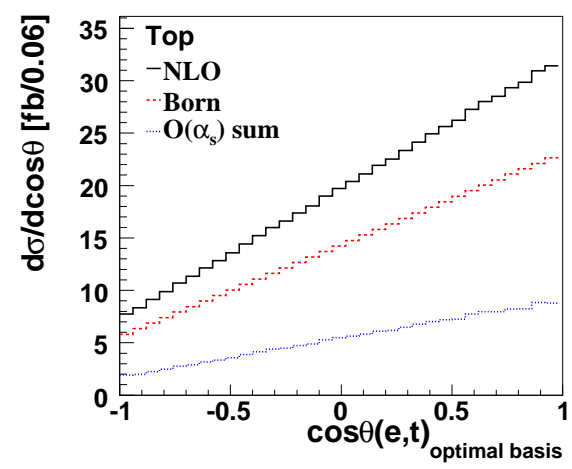

(c)

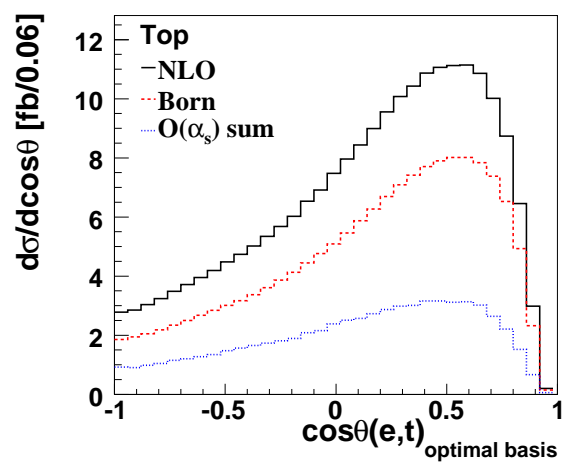

(b)

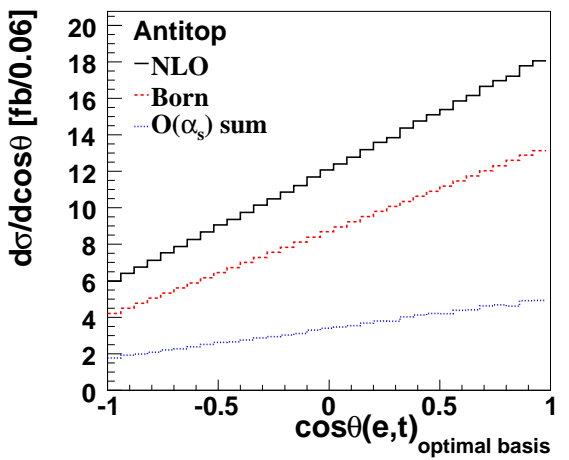

(d)

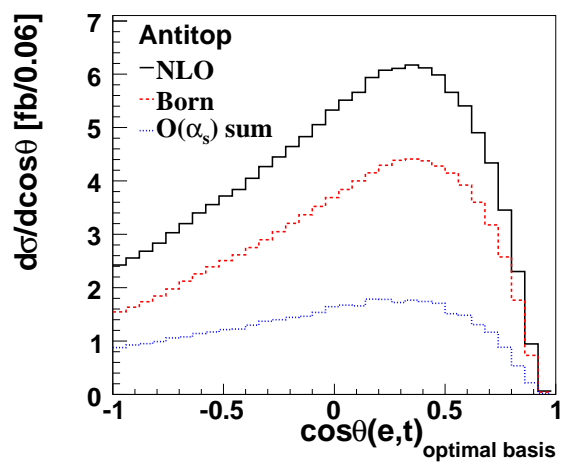

Figure 22: Top quark polarization in the optimal basis at parton level $(\mathrm{a}, \mathrm{b})$ and after event reconstruction and applying the 'loose' set of selection cuts (c, d), for top quark production (a, c) and antitop quark production (b, d).

of cuts, there is a cutoff at large $\cos \theta_{\text {opt }}$, due to the lepton $\eta$ cut.

It is possible to enhance the performance of the optimal basis, by using only those events in which $p_{z}\left(W_{\text {int }}\right)$ is larger than a certain threshold. For this study, thresholds of 500, 1000 and $2500 \mathrm{GeV}$ have been tested. By imposing a cut on $p_{z}\left(W_{i n t}\right)$, we are able to determine the direction of the incoming antiquark correctly more often, as the momentum difference between the incoming quarks increases. The efficiencies for the three different thresholds 500, 1000 and $2500 \mathrm{GeV}$ are 50\%, 25\% and 2\% respectively (cf. Fig. 18). Figure 23] shows that, at parton level, the (anti)top quark is indeed highly polarized for a $p_{z}\left(W_{\text {int }}\right)$ cut of 1000 $\mathrm{GeV}$. Reconstruction and selection cuts change the distribution dramatically and differently for top and antitop quarks. The cutoff close to $\cos \theta_{\text {opt }}=1$ is again due to the lepton $\eta$ cut. Due to spin correlations, the lepton $p_{T}$ cut carves out a large number of events with $\cos \theta_{\text {opt }}<0$ for the top quark, while for the antitop quark it removes mainly events with 
(a)

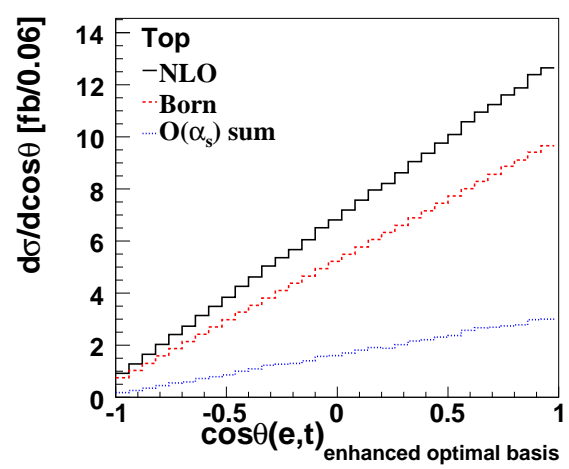

(c)

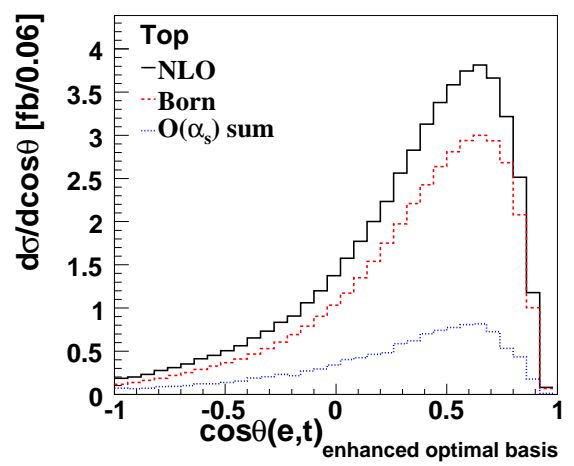

(b)

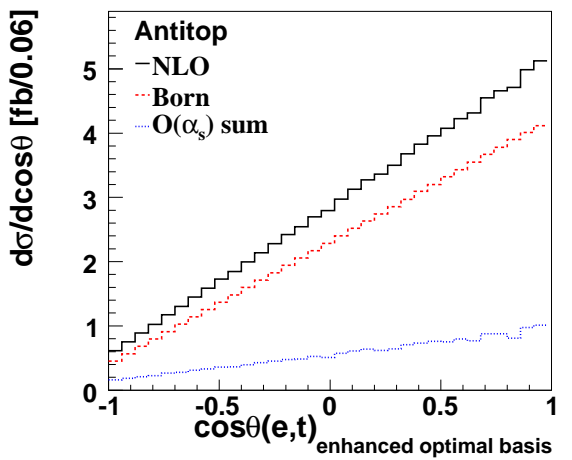

(d)

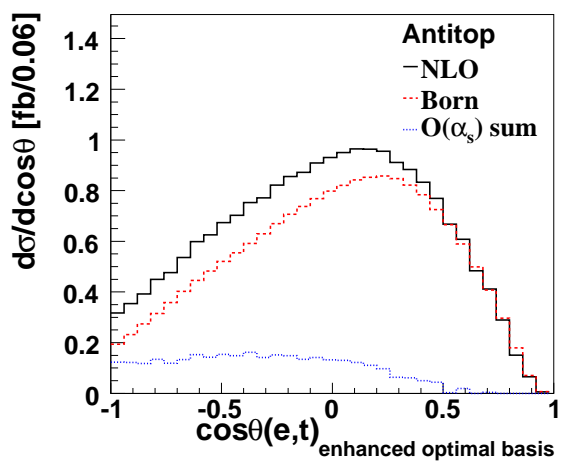

Figure 23: Top (a, c) and antitop (b, d) quark polarization in the enhanced optimal basis, where $W_{\text {int }}$ has a longitudinal momentum of at least $1000 \mathrm{GeV}$. (a, b) show the distributions before selection cuts using all parton information, while (c, d) are the distributions for reconstructed events after applying the 'loose' set of cuts.

$\cos \theta_{\text {opt }}>0$.

As in Ref. [40], we define the degree of polarization $\mathcal{D}$ of the top quark as the ratio

$$
\mathcal{D}=\frac{N_{-}-N_{+}}{N_{-}+N_{+}}
$$

where $N_{-}\left(N_{+}\right)$is the number of left-hand (right-hand) polarized top quarks in the helicity basis. Similarly, in the optimal basis, $N_{-}\left(N_{+}\right)$is the number of top quarks with polarization against (along) the direction of the chosen proton three momentum in the top quark rest frame $\vec{p}_{p_{1}}$. For the antitop quark the relationships are the same.

The spin fractions $\mathcal{F}_{ \pm}$and the asymmetry $\mathcal{A}$ of the distribution are defined in Ref. [40]. Without imposing any kinematic cuts, $D=2 \mathcal{A}$, which can indeed be seen in Table IV] Furthermore, the ratio of top quarks with spin along the basis direction will be $r_{\uparrow}=0.5-\mathcal{A}$ when no cuts are applied. However, when cuts are imposed, the two relationships break 


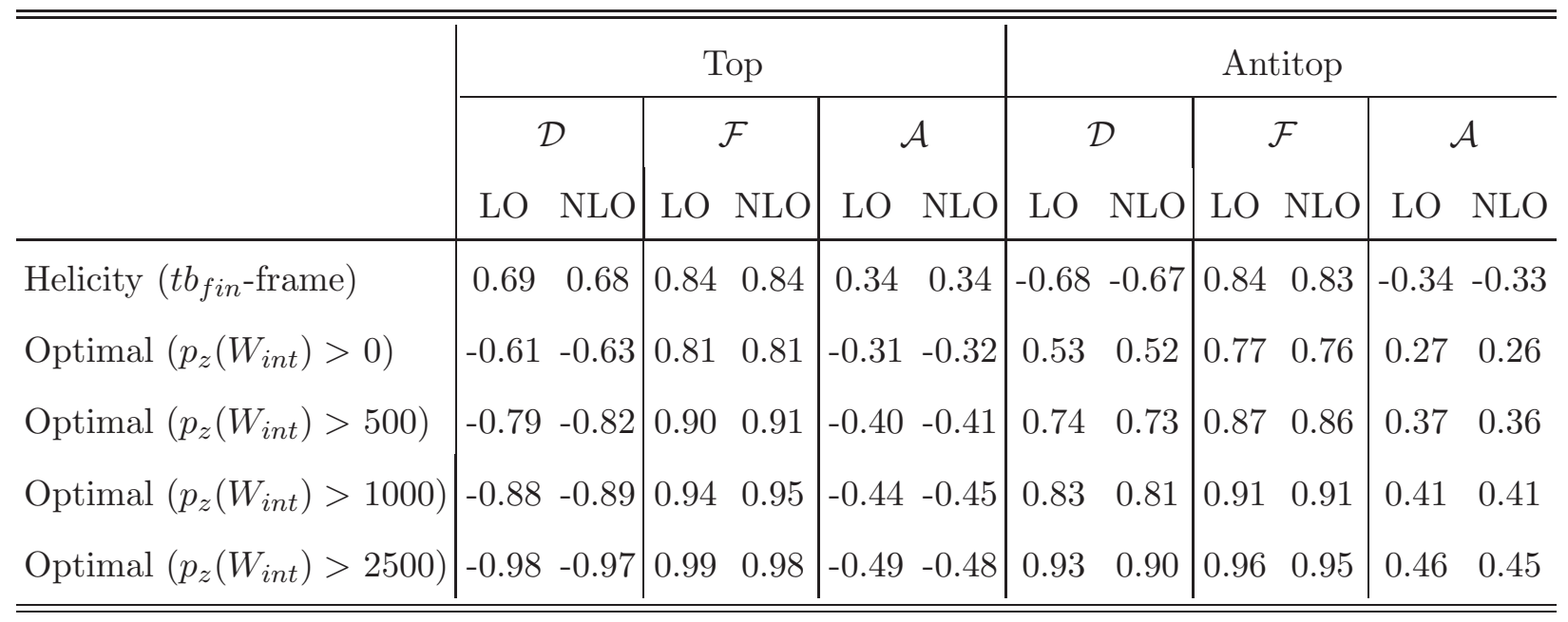

Table IV: Parton level degree of polarization $\mathcal{D}$, polarization fraction $\mathcal{F}$, and asymmetry $\mathcal{A}$ for schannel single top quark events before any cuts. Results are shown for both top (left) and antitop (right) quark measured in the helicity basis $\left(t b_{f i n}\right.$-frame) and in the optimal basis with different $p_{z}\left(W_{\text {int }}\right)$ thresholds. In this table, $\mathcal{F}$ corresponds to $\mathcal{F}_{-}$in the helicity basis for left-handed top quarks and to $\mathcal{F}_{+}$in the optimal basis for top quarks with polarization along the chosen proton three-momentum. For right-handed antitop quarks, $\mathcal{F}$ corresponds to $\mathcal{F}_{+}$in the helicity basis and to $\mathcal{F}_{-}$in the optimal basis for antitop quarks with polarization against the chosen proton three-momentum.

down.

Table IV shows $\mathcal{D}, \mathcal{F}$ and $\mathcal{A}$ at parton level before any cuts. It can be seen that in the helicity basis the polarization is slightly larger for top than for antitop quarks, as top quarks receive larger longitudinal boosts from $W_{\text {int }}$ and therefore have higher energies (cf. Sec. IV A 1). The comparison between LO and NLO shows a slight decrease in $|\mathcal{D}|$ in the helicity basis for both top and antitop quark. In the optimal basis, the larger longitudinal boost also increases the energy of the top quark, but more importantly makes it more likely that the chosen proton is the correct pick for the reference axis. As shown in Table IV] both quark types are more polarized in the helicity basis than in the optimal basis, if no $p_{z}$ cuts on $W_{\text {int }}$ are applied. With higher $p_{z}$ cuts on $W_{\text {int }},|\mathcal{D}|$ calculated in the optimal basis increases though, as higher cuts improve the chance of guessing the correct direction of the initial antiquark.

The results after reconstructing the jets $\left(p_{T}^{j} \geq 30 \mathrm{GeV},\left|\eta_{j}\right| \leq 5, \Delta R_{\ell j}=\Delta R_{j j} \geq 0.4\right)$ 


\begin{tabular}{|c|c|c|c|c|c|c|c|c|c|c|c|c|}
\hline & \multicolumn{6}{|c|}{ Top } & \multicolumn{6}{|c|}{ Antitop } \\
\hline & $\mathrm{LO}$ & $\begin{array}{l}\mathcal{D} \\
\mathrm{NLO}\end{array}$ & LO & $\begin{array}{l}\mathcal{F} \\
\mathrm{NLO}\end{array}$ & LO & $\begin{array}{l}\mathcal{A} \\
\mathrm{NLO}\end{array}$ & ${ }^{\mathcal{L}}$ & $\begin{array}{l}\mathcal{D} \\
\mathrm{NLO}\end{array}$ & $\begin{array}{r}J \\
\mathrm{LO}\end{array}$ & $\begin{array}{l}\mathcal{F} \\
\text { NLO }\end{array}$ & LO & $\begin{array}{l}\mathcal{A} \\
\mathrm{NLO}\end{array}$ \\
\hline Helicity $\left(t b_{f i n}(j)\right.$, incl. 2 -jet $)$ & 0.74 & 0.67 & 0.87 & 0.84 & 0.37 & 0.34 & -0.74 & -0.54 & 0.87 & 0.77 & -0.37 & $7-0.27$ \\
\hline Helicity $\left(t b_{f i n}\right.$, incl. 2-jet) & 0.74 & 0.72 & 0.87 & 0.86 & 0.37 & 0.36 & -0.74 & -0.71 & 0.87 & 0.86 & -0.37 & $7-0.35$ \\
\hline Helicity $\left(t b_{f i n}(j)\right.$, excl. 3-jet $)$ & & 0.76 & & 0.88 & & 0.38 & & -0.65 & & 0.83 & & -0.33 \\
\hline Helicity $\left(t b_{f i n}\right.$, excl. 3 -jet $)$ & & 0.81 & & 0.91 & & 0.41 & & -0.80 & & 0.90 & & -0.40 \\
\hline Optimal (incl. 2-jet) & -0.66 & -0.67 & 0.83 & 0.83 & -0.33 & -0.33 & 0.59 & 0.56 & 0.79 & 0.78 & 0.29 & 9.28 \\
\hline Optimal (excl. 3-jet) & & -0.69 & & 0.85 & & -0.35 & & 0.58 & & 0.79 & & 0.29 \\
\hline
\end{tabular}

Table V: Degree of polarization $\mathcal{D}$, polarization fraction $\mathcal{F}$, and asymmetry $\mathcal{A}$ for inclusive two-jet and exclusive three-jet s-channel single top quark events after jet clustering $\left(p_{T}^{j} \geq 30 \mathrm{GeV},\left|\eta_{j}\right| \leq 5\right.$, $\Delta R_{\ell j}=\Delta R_{j j} \geq 0.4$ ). Results are shown for both top (left) and antitop (right) quark measured in the helicity basis comparing the two different c.m. frames, and in the optimal basis. In this table, $\mathcal{F}$ corresponds to $\mathcal{F}_{-}$in the helicity basis for left-handed top quarks and to $\mathcal{F}_{+}$in the optimal basis for top quarks with polarization along the chosen proton three-momentum. For right-handed antitop quarks, $\mathcal{F}$ corresponds to $\mathcal{F}_{+}$in the helicity basis and to $\mathcal{F}_{-}$in the optimal basis for antitop quarks with polarization against the chosen proton three-momentum.

are listed in Table $\mathrm{V}$ for inclusive two-jet events as well as exclusive three-jet events. In comparison to the results before any cuts, $|\mathcal{D}|$ is generally larger here, as the $p_{T}$ cuts on the jets increase the energy of the (anti)top quark. Similarly, in both bases the polarization at NLO is higher for exclusive three-jet events than for inclusive two-jet events, because of the additional $p_{T}$ that a third jet in the initial state adds to the event, which again increases the energy of the (anti)top quark. Furthermore, the table shows that for the helicity basis the $t b_{f i n}$-frame leads to higher polarizations than the $t b_{f i n}(j)$-frame at NLO. This is because in most of the three-jet events the third jet comes from INIT corrections and should not be included in the c.m. system of the final state objects produced from $W_{\text {int }}$.

Figure 24 shows the degree of polarization $\mathcal{D}$ of top and antitop quark in the optimal basis, for different values of $p_{z}\left(W_{i n t}\right)$ at parton level for top and antitop quark production. 
(a)

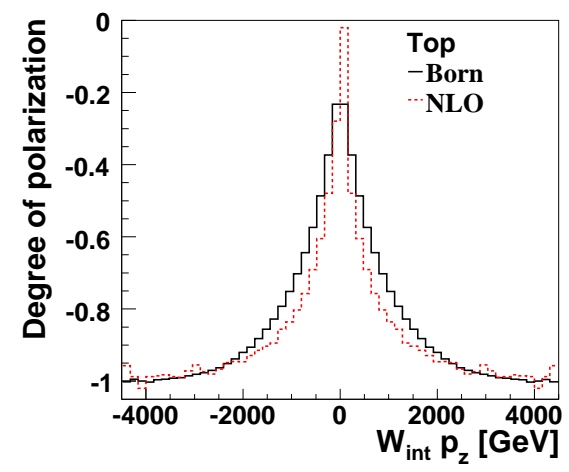

(b)

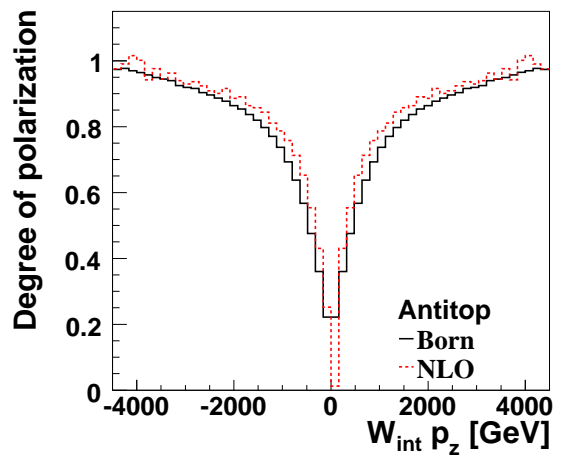

Figure 24: Degree of polarization $\mathcal{D}$ of top quark (a) and antitop quark (b) in the optimal basis, for different values of $p_{z}\left(W_{\text {int }}\right)$, at parton level before cuts.

\section{Distributions for three-jet events}

Single top quark events at the LHC contain a large fraction of three-jet events, which can be seen in Fig. 6, It is therefore of interest to discuss the kinematic properties of the third jet. After selection cuts, this third jet corresponds to $O\left(\alpha_{s}\right)$ corrections in about $80 \%$ of the three-jet events. This means, for most events, the emitted gluons/light quarks have a lower $p_{T}$ than the $b_{d e c}$ and $b_{\text {fin }}$ jets, which is mainly due to the large amount of initial state radiation that tends to be collinear to the beamline.

The emission of additional gluons/light quarks can be divided into production-stage emission and decay-stage emission. Production-state emission includes INIT and FINAL corrections and occurs before the top quark goes on-shell, while decay-stage emission consists of the SDEC contribution and occurs after the top quark goes on-shell. This classification of three-jet events into production-stage or decay-stage is useful, but blurred by the finite width of the top quark and, in experiments, by jet-energy resolution and ambiguities in jet assignment.

\section{Kinematic distributions of the extra jet}

The $p_{T}$ and $\eta$ distributions of the third jet for different NLO corrections are presented in Fig. 25. The dominance of the initial state corrections mentioned above is due to the collinear enhancement of the incoming partons and can be seen in both distributions. As it is determined by the collider energy, the INIT contribution extends to far higher $p_{T}$ and 
(a)

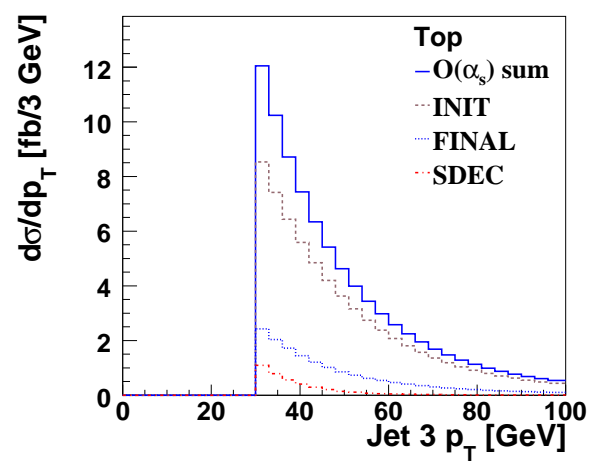

(b)

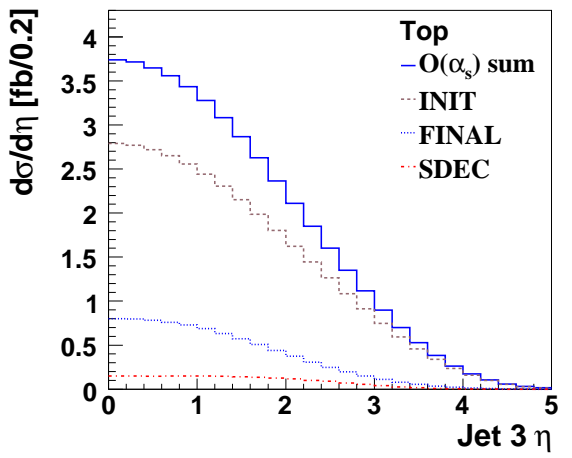

Figure 25: $p_{T}$ (a) and $\eta$ distribution (b) of the third jet after applying the 'loose' set of cuts for the different $O\left(\alpha_{s}\right)$ corrections.

(a)

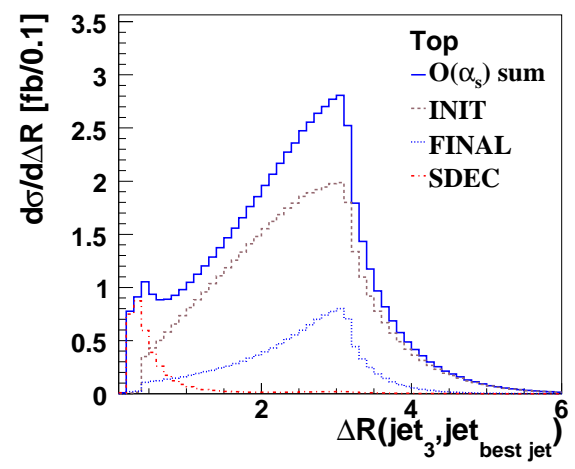

(b)

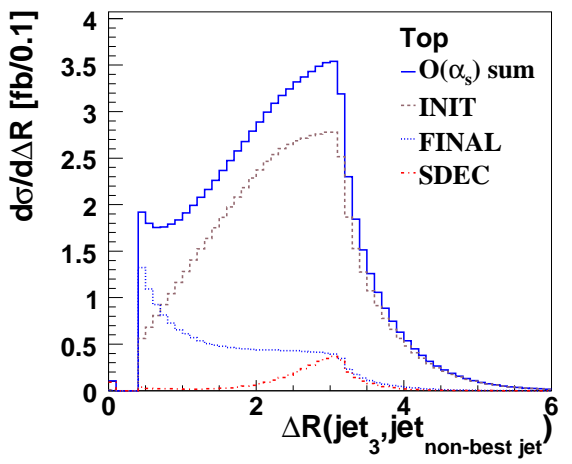

Figure 26: Difference in the $p_{T}$ between the third jet and the best-jet, if the best-jet is not the third jet (a) and the third jet and the non-best-jet (b), after applying the 'loose' set of kinematic cuts.

larger $\eta$ than the other contributions.

Figure 26 shows that it is in principle possible to identify the third jet as coming from the SDEC or the FINAL corrections, by looking at the difference in the $p_{T}$ between the third jet and the best-jet or the third jet and the non-best-jet respectively. The $p_{T}$ difference between the third jet from the decay and the best-jet has a peak close to zero for the decay contribution, while the difference between the $p_{T}$ of the third jet from the FINAL correction and the non-best-jet tends to be smaller than for the other contributions. Unfortunately those peaks are not very high, and the initial state correction is again dominant for slightly larger $p_{T}$ differences, so that an optimal cone size has to carefully balance out the competing effects of desired jets falling outside of the cone and unwanted initial state radiation being included. 


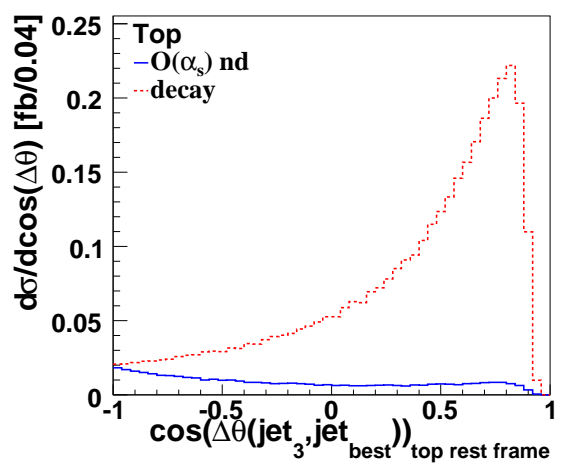

Figure 27: Angular correlation between the third jet and the best-jet after selection cuts. All NLO corrections except for SDEC (solid), denoted as $O\left(\alpha_{s}\right)$ nd, are compared to the SDEC contribution only (dotted). Here, we only allow events in which exactly two jets are used to form the best-jet.

This is further complicated in experiments by hadronization and detector resolution effects.

\section{Angular correlation between the extra jet and the best-jet}

The best-jet algorithm can be used to distinguish production-stage from decay-stage parton (gluon or quark) emission. In Fig. 27, we show the angular correlation between the third jet and the best-jet after event reconstruction. Here, we include only events in which exactly two jets are used to form the best-jet. There is a clear separation between the SDEC contribution only and the rest of the NLO corrections, even after event reconstruction with kinematic cuts imposed.

\section{CONCLUSIONS}

We have presented a study of s-channel single top and antitop quark production at the LHC based on the full NLO calculations. We have studied the NLO QCD corrections to the production and decay of the single top quark and shown their effect on the inclusive cross section as well as on kinematical distributions, including top quark polarization measurements. For this, we have divided the higher order corrections into three Gauge invariant sets. The inclusion of NLO corrections allows more precise predictions of the properties of single top quark events, which is mandatory for using single top quark events to test the 
SM and search for new physics.

The NLO corrections increase the s-channel inclusive cross section significantly. Due to the PDF of the proton, the cross section is larger for single top quark production than for single antitop quark production and depends on the value of $m_{t}$. Simple kinematic cuts, as they are used by the ATLAS and the CMS experiments, reduce the acceptance considerably, while the percentage of three-jet events remains high, especially due to collinear enhancement of the initial state corrections.

The LO kinematical distributions of final and intermediate state objects in single top quark events are dominated by spin correlations and the momentum difference between the two incoming partons. These effects are generally smaller for antitop quark production than for top quark production and reduced by $O\left(\alpha_{s}\right)$ corrections in both cases. This is because the NLO corrections weaken spin correlations and, in some cases, lower the momentum difference between the incoming partons.

The total transverse energy $H_{T}$, the invariant mass of the ( $b_{\text {dec }}$ jet, $b_{\text {fin }}$ jet $)$ system, and the invariant mass of the ( $b_{\text {dec }}$ jet, lepton) system are examples of distributions that are characteristic of single top quark events. They change significantly if NLO corrections are included. The invariant mass distribution of the SM $W_{\text {int }}$ could be a useful discriminator for $W^{\prime}$ boson searches at the LHC.

For the reconstruction of the top quark it is important to identify the correct jet as the $b_{d e c}$ jet, that is the jet which is produced when the top quark decays. The most efficient method for this identification is found to be the best-jet algorithm, which picks the $W j$ or $W j j$ combination that gives an invariant mass closest to the input $m_{t}$.

The spin correlations and the fact that the top quark decays before it can hadronize makes it possible to measure its polarization. We identify appropriate frames for top quark spin correlation measurements and find that an additional cut on $p_{z}\left(W_{\text {int }}\right)$ significantly increases the measured spin correlations in the optimal basis. 


\section{Acknowledgments}

S. H. and R. S. are supported in part by the U.S. National Science Foundation under Grant No. PHY-0757741. Q. H. C. is supported in part by the Argonne National Laboratory and University of Chicago Joint Theory Institute (JTI) Grant No. 03921-07-137, and by the U.S. Department of Energy under Grants No. DE-AC02-06CH11357 and No. DE-FG0290ER40560. C. P. Y. acknowledges the support of the U.S. National Science Foundation under Grant No. PHY-0555545 and PHY-0855561. C. P. Y. would also like to thank the hospitality of National Center for Theoretical Sciences in Taiwan and Center for High Energy Physics, Peking University, in China, where part of this work was done.

[1] D0, V. M. Abazov et al., Phys. Rev. Lett. 98, 181802 (2007), arXiv:hep-ex/0612052.

[2] D0, V. M. Abazov et al., Phys. Rev. D78, 012005 (2008), arXiv:0803.0739.

[3] CDF, T. Aaltonen et al., Phys. Rev. Lett. 101, 252001 (2008), arXiv:0809.2581.

[4] CDF, T. Aaltonen et al., Phys. Rev. Lett. 103, 092002 (2009), arXiv:0903.0885.

[5] D0, V. M. Abazov et al., Phys. Rev. Lett. 103, 092001 (2009), arXiv:0903.0850.

[6] K.-i. Hikasa, K. Whisnant, J. M. Yang, and B.-L. Young, Phys. Rev. D58, 114003 (1998), arXiv:hep-ph/9806401.

[7] T. Han, M. Hosch, K. Whisnant, B.-L. Young, and X. Zhang, Phys. Rev. D58, 073008 (1998), arXiv:hep-ph/9806486.

[8] T. Tait and C.-P. Yuan, Phys. Rev. D63, 014018 (2001), arXiv:hep-ph/0007298.

[9] J. J. Liu, C. S. Li, L. L. Yang, and L. G. Jin, Nucl. Phys. B705, 3 (2005), arXiv:hep-ph/0404099.

[10] O. Cakir and S. A. Cetin, J. Phys. G31, N1 (2005).

[11] J. Guasch, W. Hollik, S. Penaranda, and J. Sola, Nucl. Phys. Proc. Suppl. 157, 152 (2006), arXiv:hep-ph/0601218.

[12] D. O. Carlson, E. Malkawi, and C.-P. Yuan, Phys. Lett. B337, 145 (1994), arXiv:hep-ph/9405277.

[13] T. Tait and C.-P. Yuan, Phys. Rev. D55, 7300 (1997), arXiv:hep-ph/9611244.

[14] D. Espriu and J. Manzano, Phys. Rev. D65, 073005 (2002), arXiv:hep-ph/0107112. 
[15] Q.-H. Cao, C. S. Li, and C. P. Yuan, Phys. Lett. B668, 24 (2008), arXiv:hep-ph/0612243.

[16] Q.-H. Cao, J. Wudka, and C. P. Yuan, Phys. Lett. B658, 50 (2007), arXiv:0704.2809,

[17] J. A. Aguilar-Saavedra, Nucl. Phys. B804, 160 (2008), arXiv:0803.3810.

[18] D0, V. M. Abazov et al., Phys. Lett. B641, 423 (2006), arXiv:hep-ex/0607102.

[19] D0, V. M. Abazov et al., Phys. Rev. Lett. 100, 211803 (2008), arXiv:0803.3256.

[20] CDF, T. Aaltonen et al., (2009), arXiv:0902.3276.

[21] D0, V. M. Abazov et al., Phys. Rev. Lett. 99, 191802 (2007), arXiv:hep-ex/0702005.

[22] CDF, T. Aaltonen et al., Phys. Rev. Lett. 102, 151801 (2009), arXiv:0812.3400.

[23] D0, V. M. Abazov et al., Phys. Rev. Lett. 101, 221801 (2008), arXiv:0807.1692.

[24] D0, V. M. Abazov et al., Phys. Rev. Lett. 102, 092002 (2009), arXiv:0901.0151.

[25] D0, V. M. Abazov et al., Phys. Rev. Lett. 102, 191802 (2009), arXiv:0807.0859,

[26] S. Moretti, Phys. Rev. D56, 7427 (1997), arXiv:hep-ph/9705388.

[27] K. Odagiri, (1999), arXiv:hep-ph/9901432.

[28] M. C. Smith and S. Willenbrock, Phys. Rev. D54, 6696 (1996), arXiv:hep-ph/9604223.

[29] T. Stelzer and S. Willenbrock, Phys. Lett. B357, 125 (1995), arXiv:hep-ph/9505433.

[30] G. Bordes and B. van Eijk, Nucl. Phys. B435, 23 (1995).

[31] S. Zhu, (2001), arXiv:hep-ph/0109269.

[32] B. W. Harris, E. Laenen, L. Phaf, Z. Sullivan, and S. Weinzierl, Phys. Rev. D66, 054024 (2002), arXiv:hep-ph/0207055.

[33] Z. Sullivan, Phys. Rev. D70, 114012 (2004), arXiv:hep-ph/0408049.

[34] Q.-H. Cao, (2008), arXiv:0801.1539.

[35] S. Frixione, E. Laenen, P. Motylinski, B. R. Webber, and C. D. White, JHEP 07, 029 (2008), arXiv:0805.3067.

[36] J. M. Campbell, R. Frederix, F. Maltoni, and F. Tramontano, Phys. Rev. Lett. 102, 182003 (2009), arXiv:0903.0005.

[37] J. M. Campbell, R. Frederix, F. Maltoni, and F. Tramontano, (2009), arXiv:0907.3933.

[38] J. Campbell, R. K. Ellis, and F. Tramontano, Phys. Rev. D70, 094012 (2004), arXiv:hep-ph/0408158.

[39] Q.-H. Cao and C.-P. Yuan, (2004), arXiv:hep-ph/0408180.

[40] Q.-H. Cao, R. Schwienhorst, and C. P. Yuan, Phys. Rev. D71, 054023 (2005), arXiv:hep-ph/0409040. 
[41] Q.-H. Cao, R. Schwienhorst, J. A. Benitez, R. Brock, and C. P. Yuan, Phys. Rev. D72, 094027 (2005), arXiv:hep-ph/0504230

[42] S. Frixione, E. Laenen, P. Motylinski, and B. R. Webber, JHEP 03, 092 (2006), arXiv:hep-ph/0512250.

[43] S. Alioli, P. Nason, C. Oleari, and E. Re, JHEP 09, 111 (2009), arXiv:0907.4076.

[44] C. S. Li, R. J. Oakes and J. M. Yang, Phys. Rev. D55, 5780 (1997), arXiv:hep-ph/9611455.

[45] J. J. Zhang, C. S. Li, Z. Li and L. L. Yang, Phys. Rev. D75, 014020 (2007), arXiv:hep-ph/0610087.

[46] M. Beccaria, C. M. Carloni Calame, G. Macorini, E. Mirabella, F. Piccinini, F. M. Renard and C. Verzegnassi, Phys. Rev. D77, 113018 (2008), arXiv:0802.1994.

[47] J. Pumplin et al., JHEP 07, 012 (2002), arXiv:hep-ph/0201195.

[48] W. K. Tung et al., JHEP 02, 053 (2007), arXiv:hep-ph/0611254.

[49] H. L. Lai et al., JHEP 04, 089 (2007), arXiv:hep-ph/0702268.

[50] J. Pumplin, H. L. Lai, and W. K. Tung, Phys. Rev. D75, 054029 (2007), arXiv:hep-ph/0701220.

[51] P. M. Nadolsky et al., Phys. Rev. D78, 013004 (2008), arXiv:0802.0007.

[52] Q.-H. Cao and C.-P. Yuan, Phys. Rev. Lett. 93, 042001 (2004), arXiv:hep-ph/0401026.

[53] D0, V. Abazov et al., (2009), arXiv:0904.3195.

[54] W. T. Giele and E. W. N. Glover, Phys. Rev. D46, 1980 (1992).

[55] W. T. Giele, E. W. N. Glover, and D. A. Kosower, Nucl. Phys. B403, 633 (1993), arXiv:hep-ph/9302225.

[56] S. Keller and E. Laenen, Phys. Rev. D59, 114004 (1999), arXiv:hep-ph/9812415.

[57] Tevatron Electroweak Working Group, (2009), arXiv:0903.2503.

[58] UA2, J. Alitti et al., Phys. Lett. B257, 232 (1991).

[59] ATLAS, D. O’Neil, B. Gonzalez-Pineiro, and M. Lefebvre, J. Phys. G28, 2657 (2002).

[60] M. Cristinziani and G. Petrucciani, Nuovo Cim. 123B, 1142 (2008), arXiv:0808.0565.

[61] C. Kao, G. A. Ladinsky, and C. P. Yuan, (1993), arXiv:hep-ph/9305270.

[62] C.-L. Chou and M. E. Peskin, Phys. Rev. D61, 055004 (2000).

[63] G. L. Kane and C.-P. Yuan, Phys. Rev. D40, 2231 (1989).

[64] DO, B. Abbott et al., Phys. Rev. D63, 031101 (2001), arXiv:hep-ex/0008024.

[65] C.-R. Chen, F. Larios, and C. P. Yuan, (2005), arXiv:hep-ph/0503040. 
[66] G. Mahlon and S. J. Parke, Phys. Rev. D53, 4886 (1996), arXiv:hep-ph/9512264.

[67] S. J. Parke and Y. Shadmi, Phys. Lett. B387, 199 (1996), arXiv:hep-ph/9606419.

[68] G. Mahlon and S. J. Parke, Phys. Rev. D55, 7249 (1997), arXiv:hep-ph/9611367. 